\title{
Superconductivity of overdoped cuprates: the modern face of the ancestral two-electron exchange
}

\author{
T M Mishonov††, J O Indekeu $\ddagger$ and E S Penev† \\ $\dagger$ Department of Theoretical Physics, Faculty of Physics, \\ Sofia University 'St. Kliment Ohridski', \\ 5 J Bourchier Boulevard, BG-1164 Sofia, Bulgaria \\ $\ddagger$ Laboratorium voor Vaste-Stoffysica en Magnetisme, Katholieke Universiteit \\ Leuven, Celestijnenlaan 200 D, B-3001 Leuven, Belgium \\ E-mail: todor.mishonov@phys.uni-sofia.bg
}

\begin{abstract}
The single-site two-electron exchange amplitude $J_{\text {sd }}$ between the $\mathrm{Cu} 4 \mathrm{~s}$ and $\mathrm{Cu} 3 \mathrm{~d}_{x^{2}-y^{2}}$ states is found to be the pairing mechanism of high- $T_{\mathrm{C}}$ overdoped cuprates. The noninteracting part of the Hamiltonian spans the copper $\mathrm{Cu} 4 \mathrm{~s}, \mathrm{Cu} 3 \mathrm{~d}_{x^{2}-y^{2}}$ and oxygen $\mathrm{O} 2 \mathrm{p}_{x}$ and $\mathrm{O} 2 \mathrm{p}_{y}$ states. Within the standard BCS treatment an explicit expression for the momentum dependence of the gap $\Delta_{p}$ is derived and shown to fit the angle-resolved photoemission spectroscopy (ARPES) data. The basic thermodynamic and electrodynamic properties of the model [specific heat $C(T)$, London penetration depth $\lambda(T)$ ] are analytically derived. These are directly applicable to cuprates without complicating structural accessories (chains, double $\mathrm{CuO}_{2}$ planes, etc.). We advocate that the pairing mechanism of overdoped and underdoped cuprates is the same, as $T_{\mathrm{c}}$ displays smooth doping dependence. Thus, a long-standing puzzle in physics is possibly solved.

PACS numbers: 74.20.z, 74.20.Fg, 74.72.-h
\end{abstract}

\section{Introduction}

The discovery of high-temperature superconductivity [1] in cuprates and the subsequent "research rush" have led to the appearance of about 100000 papers to date [2]. Virtually every fundamental process known in condensed matter physics was probed as a possible mechanism of this phenomenon. Nevertheless, none of the theoretical efforts resulted in a coherent picture [2]. For the conventional superconductors the mechanism was known to be the interaction between electrons and crystal-lattice vibrations, but the development of its theory lagged behind the experimental findings. The case of cuprate high- $T_{\mathrm{c}}$ superconductivity appears to be the opposite: we do not convincingly know which mechanism is to be incorporated in the traditional Bardeen-Cooper-Schrieffer (BCS) theory [3]. Thus the path to high- $T_{\mathrm{c}}$ superconductivity in cuprates, perhaps carefully hidden or well-forgotten, has turned into one of the long-standing mysteries in physical science.

Features of the electronic spectrum of the $\mathrm{CuO}_{2}$ plane, figure $1(\mathrm{a})$, the structural detail responsible for the superconductivity of the cuprates, have become accessible from the angle-resolved photoemission spectroscopy (ARPES) [4,5]. Thus, any theory which pretends to explain the cuprate superconductivity is bound to include these 
features and account for them consistently. A number of extensive reviews over the past years have been devoted to that theoretical problem [6-17]. For further related discussion we also refer the reader to the review [18] on NMR-NQR spectroscopies in high- $T_{\mathrm{c}}$ superconductors.

In contrast with all previous proposals, we have advanced in reference [19] the intra-atomic exchange [20] of two electrons between the $4 \mathrm{~s}$ and $3 \mathrm{~d}_{x^{2}-y^{2}}$ states of the $\mathrm{Cu}$ atom as the origin of high- $T_{\mathrm{c}}$ superconductivity in the layered cuprates and have shown that the basic spectroscopic and thermodynamic experiments can be explained by it. Previously only inter-atomic Heitler-London-type [21] two-electron exchange [22-24] has been discussed. Thus, the present work is the unabridged version of our theory announced in reference [19]. It builds upon the standard Bloch-Hückel [25-28] (tightbinding) approximation to the electronic band structure of the $\mathrm{CuO}_{2}$ plane, developed in an earlier work [29]. We derive an analytical expression for the BCS kernel, or pairing potential $V_{p p^{\prime}}$. For the case of the s-d pairing the analytical solution is compared to the ARPES data. Extensive discussion is also provided to help the juxtaposition of our theory with other models. Finally, exact expressions within the s-d model are given for the specific heat, London penetration depth, Cooper-pair effective mass and Hall constant of the vortex-free Meissner-Ochsenfeld phase.

\section{Lattice Hamiltonian}

The electronic properties of materials are strongly influenced by the local environment and in this sense the electronic features are local physics. The simplest possible model for high- $T_{\mathrm{c}}$ superconductivity contains single-particle and correlated two-electron hoppings between nearest neighbours and next-nearest neighbours. Formally, this is an expansion of the many-particle Hamiltonian containing two- and four-fermion operators. The two-fermion Hamiltonian determines the band structure, briefly considered in subsection 2.1, while the four-fermion terms (subsection 2.2) determine the pairing interaction, and lead to the gap equations considered in section 3.

\subsection{The four-band model in a nutshell}

Every high- $T_{\mathrm{c}}$ superconductor has its specific properties. It is strongly believed, however, that the main features of the electronic band structure of the $\mathrm{CuO}_{2}$ plane are adequately described by the four-band model spanning the $\mathrm{Cu} 3 \mathrm{~d}_{x^{2}-y^{2}}, \mathrm{Cu} 4 \mathrm{~s}$, $\mathrm{O} 2 \mathrm{p}_{x}$ and $\mathrm{O} 2 \mathrm{p}_{y}$ orbitals, figure 1(b). In the spirit of the Bloch-Hückel (BH) model, using Jordan's second quantization language, we introduce Fermi annihilation operators for an electron with spin projection $\alpha$ at a particular orbital, respectively, $\hat{D}_{\boldsymbol{n} \alpha}, \hat{S}_{\boldsymbol{n} \alpha}, \hat{X}_{\boldsymbol{n} \alpha}$, and $\hat{Y}_{\boldsymbol{n} \alpha}$ in the unit cell with index $\boldsymbol{n}=\left(n_{x}, n_{y}\right)$. It is convenient to introduce a multicomponent Fermi creation operator in momentum space, $\hat{\Psi}_{p \alpha}^{\dagger}=$ $\left(\hat{D}_{\boldsymbol{p} \alpha}^{\dagger}, \hat{S}_{\boldsymbol{p} \alpha}^{\dagger}, \hat{X}_{\boldsymbol{p} \alpha}^{\dagger}, \hat{Y}_{\boldsymbol{p} \alpha}^{\dagger}\right)$. In this notation the one-electron BH Hamiltonian reads

$$
\hat{\mathcal{H}}_{\mathrm{BH}}^{\prime}=\hat{\mathcal{H}}_{\mathrm{BH}}-\mu \hat{\mathcal{N}}=\sum_{\boldsymbol{p}, \alpha} \hat{\Psi}_{\boldsymbol{p} \alpha}^{\dagger}\left(H_{\mathrm{BH}}-\mu \mathbb{1}_{4 \times 4}\right) \hat{\Psi}_{\boldsymbol{p} \alpha},
$$

where $\mu$ is the chemical potential, and (cf. reference [29])

$$
H_{\mathrm{BH}}=\left(\begin{array}{cccc}
\epsilon_{\mathrm{d}} & 0 & t_{\mathrm{pd}} s_{x} & -t_{\mathrm{pd}} s_{y} \\
0 & \epsilon_{\mathrm{s}} & t_{\mathrm{sp}} s_{x} & t_{\mathrm{sp}} s_{y} \\
t_{\mathrm{pd}} s_{x} & t_{\mathrm{sp}} s_{x} & \epsilon_{\mathrm{p}} & -t_{\mathrm{pp}} s_{x} s_{y} \\
-t_{\mathrm{pd}} s_{y} & t_{\mathrm{sp}} s_{y} & -t_{\mathrm{pp}} s_{x} s_{y} & \epsilon_{\mathrm{p}}
\end{array}\right) ;
$$


(a)

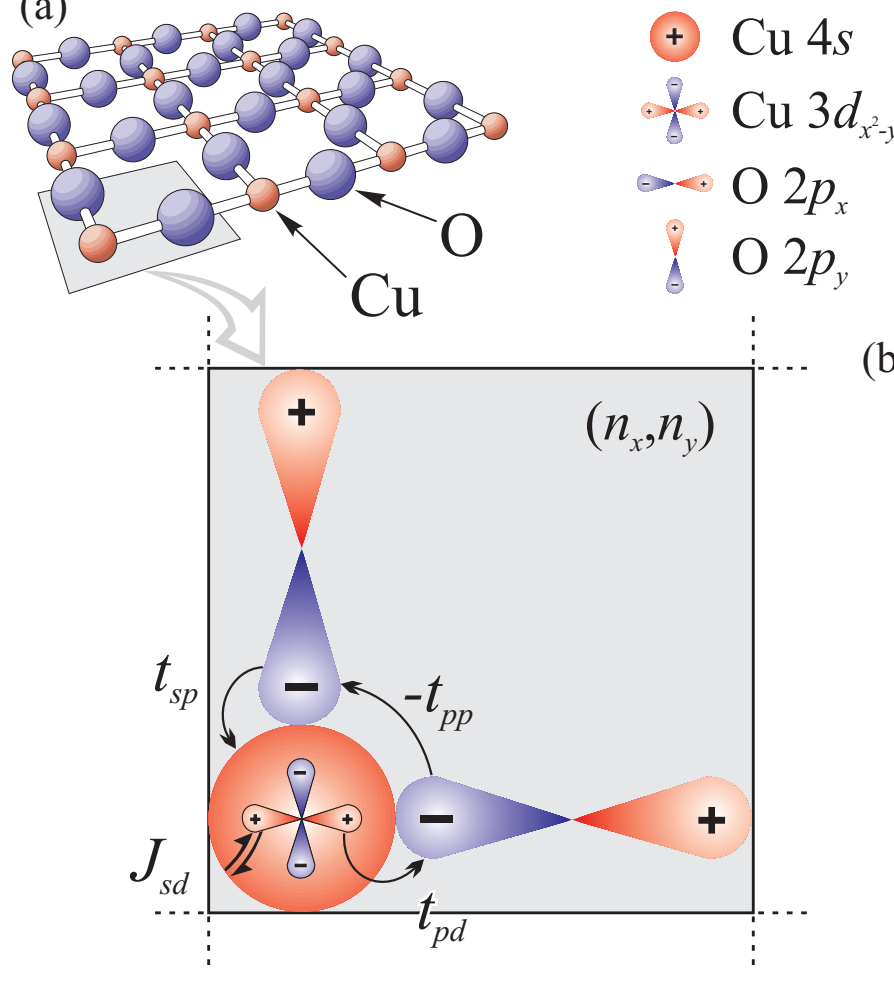

Figure 1. (a) Ball-and-stick model of the $\mathrm{CuO}_{2}$ plane. The shaded square is the unit cell indexed by $\boldsymbol{n}=\left(n_{x}, n_{y}\right), n_{x, y}=0, \pm 1, \pm 2, \ldots$ (b) The LCAO basis set: A single electron hops from the $3 \mathrm{~d}$ atomic orbital to $2 \mathrm{p}_{x}$ with amplitude $t_{\mathrm{pd}}$, contained in $\hat{\mathcal{H}}_{\mathrm{BH}}$. From $2 \mathrm{p}_{x}$ to $2 \mathrm{p}_{y}$ the hopping amplitude is $t_{\mathrm{pp}}$, and from there to $4 \mathrm{~s}$ the hopping amplitude is $t_{\mathrm{sp}}$. Correlated hopping of two electrons in opposite directions between $3 \mathrm{~d}$ and $4 \mathrm{~s}$ with amplitude $J_{\mathrm{sd}}$ is depicted as a double arrow (see the discussion in sections 4 and 5).

$\epsilon_{\mathrm{d}}, \epsilon_{\mathrm{s}}$, and $\epsilon_{\mathrm{p}}$ are the single-site energies of the $\mathrm{Cu} 3 \mathrm{~d}_{x^{2}-y^{2}}, \mathrm{Cu} 4 \mathrm{~s}, \mathrm{O} 2 \mathrm{p}_{x}$ and $\mathrm{O} 2 \mathrm{p}_{y}$ states, respectively. The hopping amplitudes between these states are $t_{\mathrm{sp}}, t_{\mathrm{pd}}$ and $t_{\mathrm{pp}}$, figure 1(b). Note, that because of the orbital orthogonality $t_{\mathrm{sd}}=0$. For brevity, we have introduced also the notation

$$
\begin{aligned}
& s_{x}=2 \sin \left(p_{x} / 2\right), \quad s_{y}=2 \sin \left(p_{y} / 2\right), \quad s=\left(s_{x}, s_{y}\right), \\
& x=\sin ^{2}\left(p_{x} / 2\right), \quad y=\sin ^{2}\left(p_{y} / 2\right) .
\end{aligned}
$$

From a classical point of view, the $\mathrm{Cu} 3 \mathrm{~d}_{x^{2}-y^{2}}$ state corresponds to a circular electron rotation in the $\mathrm{CuO}_{2}$ plane while the $\mathrm{Cu} 4 \mathrm{~s}$ state corresponds to a classical ensemble of electrons of zero angular momentum continuously falling to the nucleus. Pictorially, the s-electrons fall to the nuclei like comets, but after the impact the turning point of their motion is very far from the nucleus. This is the reason why $t_{\mathrm{sp}}$ is considerably larger than $t_{\mathrm{pd}}$. The transfer amplitude $t_{\mathrm{pp}}$ is the smallest one since the hopping to the next-nearest neighbour requires a tunnelling through free space. As a rule, the electron band calculations significantly overestimate $t_{\mathrm{pp}}$, but the latter can be reliably calculated using the surface integral method, cf. reference [29]. Even for the largest transfer integrals $t_{\mathrm{sp}}$ and $t_{\mathrm{pd}}$, which determine the bandwidth of the conduction 
band, the $a b$ initio calculations give a factor 2 or even 3 "overbinding". Nonetheless, the band calculations substantiate this choice for the LCAO (linear combination of atomic orbitals) basis set and provide an adequate language for interpretation. In the end, these parameters should be determined by fitting to the spectroscopy data and be treated in the model lattice Hamiltonian as phenomenological parameters of the microscopic many-body theory. We shall briefly recall some basic properties of the four-band model as derived in reference [29].

Let $\epsilon_{b, \boldsymbol{p}}$ and $\Psi_{b, \boldsymbol{p}}$ be the eigenvalues and the corresponding eigenvectors of the BH Hamiltonian, $H_{\mathrm{BH}} \Psi_{b, \boldsymbol{p}}=\epsilon_{b, \boldsymbol{p}} \Psi_{b, \boldsymbol{p}}$, where $b=1, \ldots, 4$ is the band index. For the "standard model", $\epsilon_{\mathrm{p}}<\epsilon_{\mathrm{d}}<\epsilon_{\mathrm{s}}$, the lowest energy band, $b=1$, is an oxygen bonding band having a minimum at the $(\pi, \pi)$ point. The next band, $b=2$, is a narrow "nonbonding" oxygen band with an exactly (within the framework of the model) zero dispersion along the $(0,0)-(\pi, 0)$ direction, i.e., this band is characterized by an extended Van Hove singularity. The conduction band, $b=3$, is a nearly halffilled $\mathrm{Cu} 3 \mathrm{~d}_{x^{2}-y^{2}}$ band with the analytical eigenvector

$\tilde{\Psi}_{3, \boldsymbol{p}}=\left(\begin{array}{c}D_{3, \boldsymbol{p}} \\ S_{3, \boldsymbol{p}} \\ X_{3, \boldsymbol{p}} \\ Y_{3, \boldsymbol{p}}\end{array}\right)=\left(\begin{array}{c}-\varepsilon_{\mathrm{s}} \varepsilon_{\mathrm{p}}^{2}+4 \varepsilon_{\mathrm{p}} t_{\mathrm{sp}}^{2}(x+y)-32 t_{\mathrm{pp}} \tau_{s p}^{2} x y \\ -4 \varepsilon_{\mathrm{p}} t_{\mathrm{sp}} t_{\mathrm{pd}}(x-y) \\ -\left(\varepsilon_{\mathrm{s}} \varepsilon_{\mathrm{p}}-8 \tau_{\mathrm{sp}}^{2} y\right) t_{\mathrm{pd}} s_{x} \\ \left(\varepsilon_{\mathrm{s}} \varepsilon_{\mathrm{p}}-8 \tau_{\mathrm{sp}}^{2} x\right) t_{\mathrm{pd}} s_{y}\end{array}\right)$,

where the $\varepsilon$ 's denote the energies measured relative to their respective atomic levels: $\varepsilon_{\mathrm{s}}=\epsilon-\epsilon_{\mathrm{s}}, \varepsilon_{\mathrm{p}}=\epsilon-\epsilon_{\mathrm{p}}, \varepsilon_{\mathrm{d}}=\epsilon-\epsilon_{\mathrm{d}}$, and $\tau_{\mathrm{sp}}^{2}=t_{\mathrm{sp}}^{2}-\varepsilon_{\mathrm{s}} t_{\mathrm{pp}} / 2$. The topmost band, $b=4$, is an empty $\mathrm{Cu} 4 \mathrm{~s}$ band. In elemental metals like $\mathrm{Cu}$ and $\mathrm{Fe}$, the $4 \mathrm{~s}$ band is a wide conduction band, but for the $\mathrm{CuO}_{2}$ plane it is completely "oxidized". Having the analytical eigenvector we can calculate the corresponding eigenvalue:

$$
\epsilon_{3, \boldsymbol{p}}=\frac{\left\langle\tilde{\Psi}_{3, \boldsymbol{p}}\left|H_{\mathrm{BH}}\right| \tilde{\Psi}_{3, \boldsymbol{p}}\right\rangle}{\left\langle\tilde{\Psi}_{3, \boldsymbol{p}} \mid \tilde{\Psi}_{3, \boldsymbol{p}}\right\rangle} .
$$

If necessary, the nonorthogonality of the atomic orbitals at neighbouring atoms can be easily taken into account. In this case the normalizing denominator in the above equation reads (for arbitrary band index)

$$
\begin{aligned}
\left\langle\tilde{\Psi}_{\boldsymbol{p}} \mid \tilde{\Psi}_{\boldsymbol{p}}\right\rangle= & D_{\boldsymbol{p}}^{2}+S_{\boldsymbol{p}}^{2}+X_{\boldsymbol{p}}^{2}+Y_{\boldsymbol{p}}^{2}+2 g_{\mathrm{pd}} s_{x} D_{\boldsymbol{p}} X_{\boldsymbol{p}}-2 g_{\mathrm{pd}} s_{y} D_{\boldsymbol{p}} Y_{\boldsymbol{p}} \\
& +2 g_{\mathrm{sp}} s_{x} S_{\boldsymbol{p}} X_{\boldsymbol{p}}+2 g_{\mathrm{sp}} s_{y} S_{\boldsymbol{p}} Y_{\boldsymbol{p}}-2 g_{\mathrm{pp}} s_{x} s_{y} X_{\boldsymbol{p}} Y_{\boldsymbol{p}}
\end{aligned}
$$

where the "metric tensor" $g_{i j}$ is given by the integral

$$
g_{i j}=\int \psi_{i}^{*}(\boldsymbol{r}) \psi_{j}(\boldsymbol{r}-\boldsymbol{R}) \mathrm{d} \boldsymbol{r},
$$

where $\psi_{i}^{*}(\boldsymbol{r})$ and $\psi_{j}(\boldsymbol{r}-\boldsymbol{R})$ are the atomic wave functions, and $\boldsymbol{R}$ is the inter-atomic distance. The phases are chosen such that all overlap integrals $g_{\mathrm{pd}}, g_{\mathrm{sp}}$, and $g_{\mathrm{pp}}$ be positive parameters, like the hopping integrals $t_{\mathrm{pd}}, t_{\mathrm{sp}}$, and $t_{\mathrm{pp}}$. Note that these provisions apply only to the single-particle spectrum. As long as one deals with a single conduction band, all Bloch states are orthogonal and the further treatment of the second-quantized Hamiltonian proceeds in the standard way.

Thus, using the Rayleigh quotient iteration for equations (2.2)-(2.5) one can obtain numerically the eigenvalue and the eigenvector. The band energies $\epsilon \equiv \epsilon_{b, \boldsymbol{p}}$ satisfy the secular equation

$$
\operatorname{det}\left(H_{\mathrm{BH}}-\epsilon \mathbb{1}_{4 \times 4}\right)=\mathcal{A} x y+\mathcal{B}(x+y)+\mathcal{C}=0,
$$


where the energy-dependent coefficients read [29]:

$$
\begin{aligned}
& \mathcal{A}(\epsilon)=16\left(4 t_{\mathrm{pd}}^{2} t_{\mathrm{sp}}^{2}+2 t_{\mathrm{sp}}^{2} t_{\mathrm{pp}} \varepsilon_{\mathrm{d}}-2 t_{\mathrm{pd}}^{2} t_{\mathrm{pp}} \varepsilon_{\mathrm{s}}-t_{\mathrm{pp}}^{2} \varepsilon_{\mathrm{d}} \varepsilon_{\mathrm{s}}\right), \\
& \mathcal{B}(\epsilon)=-4 \varepsilon_{\mathrm{p}}\left(t_{\mathrm{sp}}^{2} \varepsilon_{\mathrm{d}}+t_{\mathrm{pd}}^{2} \varepsilon_{\mathrm{s}}\right), \\
& \mathcal{C}(\epsilon)=\varepsilon_{\mathrm{d}} \varepsilon_{\mathrm{s}} \varepsilon_{\mathrm{p}}^{2} .
\end{aligned}
$$

Furthermore we introduce the normalized eigenvector $\Psi_{b, \boldsymbol{p}}=\tilde{\Psi}_{b, \boldsymbol{p}} /\left\|\tilde{\Psi}_{b, \boldsymbol{p}}\right\|$ and write the noninteracting Hamiltonian in diagonal form,

$$
\hat{\mathcal{H}}_{\mathrm{BH}}^{\prime}=\sum_{b, \boldsymbol{p}, \alpha}\left(\epsilon_{b, \boldsymbol{p}}-\mu\right) \hat{c}_{b, \boldsymbol{p} \alpha}^{\dagger} \hat{c}_{b, \boldsymbol{p} \alpha} .
$$

The Fermi operators in real-space representation can be easily expressed using the band representation,

$$
\hat{\Psi}_{\boldsymbol{n} \alpha} \equiv\left(\begin{array}{c}
\hat{D}_{\boldsymbol{n} \alpha} \\
\hat{S}_{\boldsymbol{n} \alpha} \\
\hat{X}_{\boldsymbol{n} \alpha} \\
\hat{Y}_{\boldsymbol{n} \alpha}
\end{array}\right)=\frac{1}{\sqrt{N}} \sum_{b, \boldsymbol{p}} \mathrm{e}^{\mathrm{i} \boldsymbol{p} \cdot \boldsymbol{n}}\left(\begin{array}{c}
D_{b, \boldsymbol{p}} \\
S_{b, \boldsymbol{p}} \\
\mathrm{e}^{\mathrm{i} \varphi_{x}} X_{b, \boldsymbol{p}} \\
\mathrm{e}^{\mathrm{i} \varphi_{y} Y_{b, \boldsymbol{p}}}
\end{array}\right) \hat{c}_{b, \boldsymbol{p} \alpha},
$$

where $N$ is the number of unit cells, and the two phases in the right-hand side of the equation read $\varphi_{x}=\frac{1}{2}\left(p_{x}-\pi\right)$ and $\varphi_{y}=\frac{1}{2}\left(p_{y}-\pi\right)$. This transformation will be used in the next subsection for deriving the interaction Hamiltonian.

\subsection{The Heitler-London \& Schubin-Wonsowsky-Zener interaction}

The Heitler-London (HL) interaction Hamiltonian describes the (intra- and interatomic) two-electron exchange. It comprises four parts [22,23] corresponding to $\mathrm{Cu} 4 \mathrm{~s} \leftrightarrow \mathrm{O} 2 \mathrm{p} \sigma, \mathrm{O} 2 \mathrm{p} \sigma \leftrightarrow \mathrm{Cu} 3 \mathrm{~d}_{x^{2}-y^{2}}, \mathrm{O} 2 \mathrm{p}_{x} \leftrightarrow \mathrm{O} 2 \mathrm{p}_{y}$, and $\mathrm{Cu} 3 \mathrm{~d}_{x^{2}-y^{2}} \leftrightarrow \mathrm{Cu} 4 \mathrm{~s}$ exchanges with transition amplitudes $J_{\mathrm{sp}}, J_{\mathrm{pd}}, J_{\mathrm{pp}}$, and $J_{\mathrm{sd}}$, respectively:

$$
\begin{aligned}
& \hat{\mathcal{H}}_{\mathrm{HL}}=- J_{\mathrm{sd}} \\
& \sum_{\boldsymbol{n}, \alpha \beta} \hat{S}_{\boldsymbol{n} \alpha}^{\dagger} \hat{D}_{\boldsymbol{n} \beta}^{\dagger} \hat{S}_{\boldsymbol{n} \beta} \hat{D}_{\boldsymbol{n} \alpha} \\
&-J_{\mathrm{sp}} \sum_{\boldsymbol{n}, \alpha \beta}\left[\hat{S}_{\boldsymbol{n} \alpha}^{\dagger} \hat{X}_{\boldsymbol{n} \beta}^{\dagger} \hat{S}_{\boldsymbol{n} \beta} \hat{X}_{\boldsymbol{n} \alpha}+\hat{S}_{\boldsymbol{n} \alpha}^{\dagger} \hat{Y}_{\boldsymbol{n} \beta}^{\dagger} \hat{S}_{\boldsymbol{n} \beta} \hat{Y}_{\boldsymbol{n} \alpha}\right. \\
&\left.\quad+\hat{S}_{\left(n_{x}+1, n_{y}\right) \alpha}^{\dagger} \hat{X}_{\boldsymbol{n} \beta}^{\dagger} \hat{S}_{\left(n_{x}+1, n_{y}\right) \beta} \hat{X}_{\boldsymbol{n} \alpha}+\hat{S}_{\left(n_{x}, n_{y}+1\right) \alpha}^{\dagger} \hat{Y}_{\boldsymbol{n} \beta}^{\dagger} \hat{S}_{\left(n_{x}, n_{y}+1\right) \beta} \hat{Y}_{\boldsymbol{n} \alpha}\right] \\
&-J_{\mathrm{pd}} \sum_{\boldsymbol{n}, \alpha \beta}\left[\hat{D}_{\boldsymbol{n} \alpha}^{\dagger} \hat{X}_{\boldsymbol{n} \beta}^{\dagger} \hat{D}_{\boldsymbol{n} \beta} \hat{X}_{\boldsymbol{n} \alpha}+\hat{D}_{\boldsymbol{n} \alpha}^{\dagger} \hat{Y}_{\boldsymbol{n} \beta}^{\dagger} \hat{D}_{\boldsymbol{n} \beta} \hat{Y}_{\boldsymbol{n} \alpha}\right. \\
&\left.\quad+\hat{D}_{\left(n_{x}+1, n_{y}\right) \alpha}^{\dagger} \hat{X}_{\boldsymbol{n} \beta}^{\dagger} \hat{D}_{\left(n_{x}+1, n_{y}\right) \beta} \hat{X}_{\boldsymbol{n} \alpha}+\hat{D}_{\left(n_{x}, n_{y}+1\right) \alpha}^{\dagger} \hat{Y}_{\boldsymbol{n} \beta}^{\dagger} \hat{D}_{\left(n_{x}, n_{y}+1\right) \beta} \hat{Y}_{\boldsymbol{n} \alpha}\right] \\
&-J_{\mathrm{pp}} \sum_{\boldsymbol{n}, \alpha \beta}\left[\hat{X}_{\boldsymbol{n} \alpha}^{\dagger} \hat{Y}_{\boldsymbol{n} \beta}^{\dagger} \hat{X}_{\boldsymbol{n} \beta} \hat{Y}_{\boldsymbol{n} \alpha}+\hat{X}_{\boldsymbol{n} \alpha}^{\dagger} \hat{Y}_{\left(n_{x}+1, n_{y}\right) \beta}^{\dagger} \hat{X}_{\boldsymbol{n} \beta} \hat{Y}_{\left(n_{x}+1, n_{y}\right) \alpha}\right. \\
& \quad+\hat{X}_{\left(n_{x}, n_{y}+1\right) \alpha}^{\dagger} \hat{Y}_{\boldsymbol{n} \beta}^{\dagger} \hat{X}_{\left(n_{x}, n_{y}+1\right) \beta} \hat{Y}_{\boldsymbol{n} \alpha} \\
&\left.+\hat{X}_{\left(n_{x}, n_{y}+1\right) \alpha}^{\dagger} \hat{Y}_{\left(n_{x}+1, n_{y}\right) \beta}^{\dagger} \hat{X}_{\left(n_{x}, n_{y}+1\right) \beta} \hat{Y}_{\left(n_{x}+1, n_{y}\right) \alpha}\right] .
\end{aligned}
$$

Let us now analyze the structure of the total electron Hamiltonian $\hat{\mathcal{H}}^{\prime}=\hat{\mathcal{H}}_{\mathrm{BH}}^{\prime}+\hat{\mathcal{H}}_{\mathrm{HL}}$. In terms of the Fermi operators $\hat{\Psi}_{i \alpha}$, corresponding to the atomic orbitals, $\hat{\mathcal{H}}^{\prime}$ reads:

$$
\hat{\mathcal{H}}^{\prime}=\sum_{i, \alpha}\left(\epsilon_{i}-\mu\right) \hat{\Psi}_{i \alpha}^{\dagger} \hat{\Psi}_{i \alpha}-\sum_{i<j, \alpha}\left(\tilde{t}_{j i} \hat{\Psi}_{j \alpha}^{\dagger} \hat{\Psi}_{i \alpha}+\tilde{t}_{j i}^{*} \hat{\Psi}_{i \alpha}^{\dagger} \hat{\Psi}_{j \alpha}\right)
$$




$$
-\sum_{i<j, \alpha \beta} J_{i j} \hat{\Psi}_{i \beta}^{\dagger} \hat{\Psi}_{j \alpha}^{\dagger} \hat{\Psi}_{i \alpha} \hat{\Psi}_{j \beta},
$$

where $\tilde{t}_{j i}=t_{j i} \mathrm{e}^{\mathrm{i} \phi_{j i}}, t_{j i}=t_{i j}$ and $\phi_{j i}=\phi_{j}-\phi_{i}$ is the phase difference between the $i$ th and $j$ th atomic orbitals in the overlapping domain. Roughly speaking, onto every single-electron hopping amplitude $t_{i j}$ one can map a corresponding two-electron hopping amplitude $J_{i j}$. The case of a strong electron correlation implies that $J_{i j}$ could be of the order of $t_{i j}$. Thus, one can expect that the following inequalities hold true $J_{\mathrm{pp}}<J_{\mathrm{pd}}<J_{\mathrm{sp}}<J_{\mathrm{sd}}$.

In fact, the s-d exchange is the basic process responsible for the magnetism of transition metals; see for example reference [20]. It was understood since the dawn of quantum physics that the mechanism of ferromagnetism [30] is the two-electron exchange owing to the electron correlations [31].

Here we shall add a few words in retrospect concerning the two-electron correlation parameterized by $J_{i j}$ in (2.13). Probably the first two-electron problem was Bohr's consideration of the He atom [32] (cf. references [33,34]) in which two electrons have opposite coordinates $\boldsymbol{r}_{2}=-\boldsymbol{r}_{1}$ and momenta $\boldsymbol{p}_{2}=-\boldsymbol{p}_{1}$. For a purely radial motion, such a fall to the nucleus is stable and many years after Bohr's prediction double Rydberg states, with an effective $\mathrm{Ry}_{\text {eff }}=(2-1 / 4) \mathrm{Ry}$, were discovered by electron energy loss spectroscopy [35]. These double Rydberg states with opposite electron momenta can be considered as proto-forms of the Cooper pairs. Interestingly, in 1914, Sir J J Thomson proposed [36] (cf. also the textbook [37]) that electric charge can propagate as electron doublets - another proto-form of the local (OggSchafroth) pairs [38,39]. Before the appearance of quantum mechanics, Lewis [40] and Langmuir [41] introduced the idea of electron doublets in order to explain the nature of the chemical bond. Nearly at the same time Parson [42] came to the conclusion that "an electron is not merely an electron charge but a small magnet" or in his terminology "a magneton", cf. reference [40]. Later, in 1926, Lewis introduced also the notion of a photon [43] without any reliable theoretical background at the time.

In the era of new quantum mechanics Heitler and London [21] realized the idea of electron doublets [44] and convincingly demonstrated how the two-particle correlation owing to a strong Coulomb repulsion can lead to a decrease of the energy, and by virtue of the Hellmann-Feynman theorem, to inter-atomic attraction for the singlet state of the electron doublet. The original Heitler-London calculation, which is nowadays interpreted in every textbook in quantum mechanics and/or quantum chemistry, gives indeed a wrong sign of the exchange energy for very large inter-atomic distances but, in principle, there are no conceptual difficulties in the Heitler-London theory. The exchange energy $J$ was represented [45] as a surface integral in the two-electron sixdimensional space $\left(\boldsymbol{r}_{1}, \boldsymbol{r}_{2}\right)$ and this was shown to be an asymptotically exact result, cf. also reference [46]. The surface integral method gives amazingly accurate results (cf. the excellent monograph by Patil and Tang [47]) even if the exchange energy is of the order of the energies typical for solid state phenomena. Unfortunately, this method, that ought to be applied to ab initio calculated (e.g., from density functional theory (DFT) [48]) wave functions, is barely known in the solid state community (although a very recent work by Gor'kov and Krotkov [49] indicates that it is not completely forgotten).

This is one of the reasons why the $t$ and $J$ transfer integrals have been treated phenomenologically just as fitting parameters of the theory. A valuable discussion on a similar scope of ideas has recently been given by Brovetto, Maxia and Salis [50] but 
it may well not be the only case. In order to ease comparison of the HL Hamiltonian with the other types discussed in the search of a theory of high- $T_{\mathrm{c}}$ superconductivity we shall rewrite it in terms of spin variables.

The grounds for our theory have been set first by Schubin and Wonsowsky and later in more clear notions and notation by Zener [20]. The s-d two-electron exchange is the intra-atomic version of the HL interaction. Both of those 4-fermion interactions due to Heitler-London \& Schubin-Wonsowsky-Zener can in principle mediate superconductivity and magnetism.

2.2.1. Spin variables Let us introduce the spin operator $\hat{\boldsymbol{S}}_{i}$ and particle number operator $\hat{n}_{i}$ for each atomic orbital,

$$
\hat{S}_{i}=\hat{\Psi}_{i}^{\dagger} \frac{\sigma}{2} \hat{\Psi}_{i}, \quad \hat{n}_{i}=\hat{\Psi}_{i}^{\dagger} \sigma_{0} \hat{\Psi}_{i}, \quad \hat{\Psi}_{i}^{\dagger}=\left(\hat{\Psi}_{i \uparrow}^{\dagger}, \hat{\Psi}_{i \downarrow}^{\dagger}\right),
$$

where $\sigma_{0}=\mathbb{1}_{2 \times 2}$ and $\sigma$ are the Pauli sigma matrices, and the first two formulae imply summation over the spin indices. Introducing also the spin exchange operator $\hat{P}_{i j}$,

$$
P \hat{\Psi}_{i \alpha} \hat{\Psi}_{j \beta}=\hat{\Psi}_{i \beta} \hat{\Psi}_{j \alpha}, \quad \hat{P}_{i j}=\sum_{\alpha \beta}\left(\hat{\Psi}_{i \alpha} \hat{\Psi}_{j \beta}\right)^{\dagger} P \hat{\Psi}_{i \alpha} \hat{\Psi}_{j \beta},
$$

we can rewrite the HL Hamiltonian per bond as [51-53]

$$
-J \sum_{\alpha \beta} \hat{\Psi}_{i \beta}^{\dagger} \hat{\Psi}_{j \alpha}^{\dagger} \hat{\Psi}_{i \alpha} \hat{\Psi}_{j \beta}=J \hat{P}_{i j}=2 J\left(\hat{\boldsymbol{S}}_{i} \cdot \hat{\boldsymbol{S}}_{j}+\frac{1}{4} \hat{n}_{i} \hat{n}_{j}\right) .
$$

We should stress that in the $t$ - $J$ model the term $\propto \hat{n}_{i} \hat{n}_{j}$ enters with negative sign $[54,56]$. Let us also provide the "mixed" representation:

$$
\begin{aligned}
2 \hat{\boldsymbol{S}}_{i} \cdot \hat{\boldsymbol{S}}_{j}= & \hat{S}_{i, x}\left(\hat{\Psi}_{j \uparrow}^{\dagger} \hat{\Psi}_{j \downarrow}+\hat{\Psi}_{j \downarrow}^{\dagger} \hat{\Psi}_{j \uparrow}\right) \\
& +\hat{S}_{i, y}\left(-i \hat{\Psi}_{j \uparrow}^{\dagger} \hat{\Psi}_{j \downarrow}+i \hat{\Psi}_{j \downarrow}^{\dagger} \hat{\Psi}_{j \uparrow}\right)+\hat{S}_{i, z}\left(\hat{n}_{j \uparrow}-\hat{n}_{j \downarrow}\right) \\
= & \hat{S}_{i,+} \hat{\Psi}_{j \downarrow}^{\dagger} \hat{\Psi}_{j \uparrow}+\hat{S}_{i,-} \hat{\Psi}_{j \uparrow}^{\dagger} \hat{\Psi}_{j \downarrow}+\hat{S}_{i, z}\left(\hat{n}_{j \uparrow}-\hat{n}_{j \downarrow}\right),
\end{aligned}
$$

where $\hat{n}_{j \uparrow} \equiv \hat{\Psi}_{j \uparrow}^{\dagger} \hat{\Psi}_{j \uparrow}$, and $\hat{S}_{i,+}=\hat{\Psi}_{i \uparrow}^{\dagger} \hat{\Psi}_{i \downarrow}=\hat{S}_{i,-}^{\dagger}$. Note that (2.16) implies a purely orbital motion without spin flip: two electrons exchange their orbitals and only the spin indices reflect this correlated hopping. For $J>0$, the HL Hamiltonian has a singlet ground state

$|\mathrm{S}\rangle=\frac{1}{\sqrt{2}}\left(\hat{\Psi}_{i \uparrow}^{\dagger} \hat{\Psi}_{j \downarrow}^{\dagger}-\hat{\Psi}_{i \downarrow}^{\dagger} \hat{\Psi}_{j \uparrow}^{\dagger}\right)|\mathrm{vac}\rangle, \quad \hat{\Psi}_{i \alpha}|\mathrm{vac}\rangle=0, \quad\langle\operatorname{vac} \mid \mathrm{vac}\rangle=1$,

with eigenvalue $-J$. The lowering in energy of the singlet state, having a symmetric orbital wave function, is of purely kinetic origin related to the delocalization of the particles at different orbitals. Symbolically, the "location" of the (approximately) localized electron doublet(s) in the structure signature of a molecule is designated by a colon, e.g., $\mathrm{H}: \mathrm{H}$ for the $\mathrm{H}_{2}$ molecule. This Lewis notation for the valence bond with energy $-J$ (or four-Fermion terms in the second quantization language) is an important ingredient of the chemical intuition. In principle, such an exchange lowering is expected to exist for Bose particles as well. For electrons, however, we have triplet excited states

$$
\begin{aligned}
& |\mathrm{T}+1\rangle=\hat{\Psi}_{i \uparrow}^{\dagger} \hat{\Psi}_{j \uparrow}^{\dagger}|\mathrm{vac}\rangle, \\
& |\mathrm{T} 0\rangle=\frac{1}{\sqrt{2}}\left(\hat{\Psi}_{i \uparrow}^{\dagger} \hat{\Psi}_{j \downarrow}^{\dagger}+\hat{\Psi}_{i \downarrow}^{\dagger} \hat{\Psi}_{j \uparrow}^{\dagger}\right)|\mathrm{vac}\rangle, \\
& |\mathrm{T}-1\rangle=\hat{\Psi}_{i \downarrow}^{\dagger} \hat{\Psi}_{j \downarrow}^{\dagger}|\mathrm{vac}\rangle,
\end{aligned}
$$


with eigenvalue $J$. In the present work we consider the parameter $J$ to be positive if it corresponds to antiferromagnetism, or pairing in the singlet channel. Thus the singlet-triplet splitting for the single-bond HL Hamiltonian (2.16) is $2 J$. Similarly, the bonding-antibonding splitting for the single-particle hopping Hamiltonian $-t \sum_{\alpha}\left(\hat{\Psi}_{j \alpha}^{\dagger} \hat{\Psi}_{i \alpha}+\hat{\Psi}_{i \alpha}^{\dagger} \hat{\Psi}_{j \alpha}\right)$ is $2 t$, and the energy threshold for creation of a pair of normal carriers, considered in the next section, is $2 \Delta$. Besides stemming from bare inter- and intra-atomic processes, two-electron hopping amplitudes $J$ can be created by strong correlations [55] within the Hubbard model. For a nice review on this subject the reader is referred to the work by Spalek and Honig [56].

\section{Reduced Hamiltonian and the BCS gap equation}

Substituting the Fermi operators $\hat{\Psi}_{\boldsymbol{n} \alpha}$, equation (2.11), into equation (2.12) one obtains the HL interaction Hamiltonian in a diagonal band representation. For the case of zero electric current [57], solely the reduced Hamiltonian $\hat{\mathcal{H}}_{\mathrm{HL}-\mathrm{R}}$, including creation and annihilation operators with opposite momenta only, has to be taken into account:

$$
\hat{\mathcal{H}}_{\mathrm{HL}-\mathrm{R}}=\frac{1}{2 N} \sum_{b, \boldsymbol{p}} \sum_{b^{\prime}, \boldsymbol{p}^{\prime}} \sum_{\alpha \beta} \hat{c}_{b, \boldsymbol{p} \beta}^{\dagger} \hat{c}_{b,-\boldsymbol{p} \alpha}^{\dagger} V_{b, \boldsymbol{p} ; b^{\prime}, \boldsymbol{p}^{\prime}} \hat{c}_{b^{\prime},-\boldsymbol{p}^{\prime} \alpha} \hat{c}_{b^{\prime}, \boldsymbol{p}^{\prime} \beta} .
$$

For singlet superconductors it is necessary to take into account the pairing with opposite spins, thereby the total reduced Hamiltonian reads

$\hat{\mathcal{H}}_{\mathrm{R}}^{\prime}=\sum_{b, \boldsymbol{p}, \alpha} \eta_{b, \boldsymbol{p}} \hat{c}_{b, \boldsymbol{p} \alpha}^{\dagger} \hat{c}_{b, \boldsymbol{p} \alpha}+\frac{1}{N} \sum_{b, \boldsymbol{p}} \sum_{b^{\prime}, \boldsymbol{p}^{\prime}} V_{b, \boldsymbol{p} ; b^{\prime}, \boldsymbol{p}^{\prime}} \hat{c}_{b, \boldsymbol{p} \uparrow}^{\dagger} \hat{c}_{b,-\boldsymbol{p} \downarrow}^{\dagger} \hat{c}_{b^{\prime},-\boldsymbol{p}^{\prime} \downarrow} \hat{c}_{b^{\prime}, \boldsymbol{p}^{\prime} \uparrow}$,

where $\eta_{b, \boldsymbol{p}} \equiv \epsilon_{b, \boldsymbol{p}}-\mu$ are the band energies measured from the chemical potential [57]. Hence the BCS equation [3] for the superconducting gap takes the familiar form

$$
\Delta_{b, \boldsymbol{p}}=\frac{1}{N} \sum_{b^{\prime}, \boldsymbol{p}^{\prime}}\left(-V_{b, \boldsymbol{p} ; b^{\prime}, \boldsymbol{p}^{\prime}}\right) \frac{1-2 n_{b^{\prime}, \boldsymbol{p}^{\prime}}}{2 E_{b^{\prime}, \boldsymbol{p}^{\prime}}} \Delta_{b^{\prime}, \boldsymbol{p}^{\prime}}
$$

where $E_{b, \boldsymbol{p}}=\left(\eta_{b, \boldsymbol{p}}^{2}+\left|\Delta_{b, \boldsymbol{p}}\right|^{2}\right)^{1 / 2}$ are the quasiparticle energies and $n_{b, \boldsymbol{p}}=$ $\left[\exp \left(E_{b, p} / k_{\mathrm{B}} T\right)+1\right]^{-1}$ are the Fermi filling factors with $k_{\mathrm{B}}$ the Boltzmann constant, and $T$ the temperature. The summation over the band index $b^{\prime}$ should be restricted to the partially filled (metallic) bands, comprising sheets of the Fermi surface. Applying this standard procedure to the HL Hamiltonian (2.12), and after some algebra, we obtain the desired BCS pairing kernel,

$$
\begin{aligned}
V_{b, \boldsymbol{p} ; b^{\prime}, \boldsymbol{p}^{\prime}}= & -2 J_{\mathrm{sd}} S_{\boldsymbol{p}} S_{\boldsymbol{p}^{\prime}} D_{\boldsymbol{p}} D_{\boldsymbol{p}^{\prime}}-J_{\mathrm{pp}} \gamma_{x} X_{\boldsymbol{p}} X_{\boldsymbol{p}^{\prime}} \gamma_{y} Y_{\boldsymbol{p}} Y_{\boldsymbol{p}^{\prime}} \\
& +2\left(J_{\mathrm{sp}} S_{\boldsymbol{p}} S_{\boldsymbol{p}^{\prime}}+J_{\mathrm{pd}} D_{\boldsymbol{p}} D_{\boldsymbol{p}^{\prime}}\right)\left(\gamma_{x} X_{\boldsymbol{p}} X_{\boldsymbol{p}^{\prime}}+\gamma_{y} Y_{\boldsymbol{p}} Y_{\boldsymbol{p}^{\prime}}\right),
\end{aligned}
$$

where

$$
\gamma_{x}=4 \cos \left(\frac{p_{x}+p_{x}^{\prime}}{2}\right), \quad \gamma_{y}=4 \cos \left(\frac{p_{y}+p_{y}^{\prime}}{2}\right) .
$$

As the band indices $b$ and $b^{\prime}$ enter implicitly in the band energies $\epsilon_{b, p}$ in the equation for the eigenvectors $\Psi_{\boldsymbol{p}}\left(\epsilon_{b, \boldsymbol{p}}\right)$, we will suppress them hereafter. The layered cuprates, admittedly, have a single conduction band and their Fermi surface has the shape of 
a rounded square. In this simplest case one has to solve numerically the nonlinear integral equation

$$
\Delta_{\boldsymbol{p}}=\int_{-\pi}^{\pi} \frac{\mathrm{d} q_{x}}{2 \pi} \int_{-\pi}^{\pi} \frac{\mathrm{d} q_{y}}{2 \pi}\left(-V_{\boldsymbol{p} \boldsymbol{q}}\right) \frac{\Delta_{\boldsymbol{q}}}{2 E_{\boldsymbol{q}}} \tanh \left(\frac{E_{\boldsymbol{q}}}{2 k_{\mathrm{B}} T}\right) .
$$

The solution to this general gap equation, depending on the $J_{i j}$ values, can exhibit s, p, or d-type symmetry. It has been shown previously that a purely p-p model $[23,58]$ $\left(J_{\mathrm{pp}}>0\right)$ results in a $\mathrm{d}_{x y}\left(B_{2 g}\right)$ gap anisotropy. However, we found that an agreement with the experimentally observed $\mathrm{d}_{x^{2}-y^{2}}\left(B_{1 g}\right)$ gap anisotropy (for a review see for example reference [59]) can be achieved only in the simplest possible case of a dominant s-d exchange. This separable Hamiltonian deserves special attention and we will analyze it in the next sections.

\section{Separable s-d model}

For the special case of a purely s-d model, $J_{\mathrm{sp}}=J_{\mathrm{pd}}=J_{\mathrm{pp}}=0$, representing the spin exchange operator $\hat{P}$ as a $(4 \times 4)$-matrix, cf. equations $(2.15)-(2.16)$, the reduced pairing Hamiltonian takes the form

$\hat{\mathcal{H}}_{\mathrm{HL}-\mathrm{R}}=\frac{J_{\mathrm{Sd}}}{N} \sum_{\boldsymbol{p}, \boldsymbol{q}}\left(\begin{array}{c}\hat{S}_{-\boldsymbol{p} \uparrow} \hat{D}_{\boldsymbol{p} \uparrow} \\ \hat{S}_{-\boldsymbol{p} \uparrow} \hat{D}_{\boldsymbol{p} \downarrow} \\ \hat{S}_{-\boldsymbol{p} \downarrow} \hat{D}_{\boldsymbol{p} \uparrow} \\ \hat{S}_{-\boldsymbol{p} \downarrow} \hat{D}_{\boldsymbol{p} \downarrow}\end{array}\right)^{\dagger}\left(\begin{array}{cccc}1 & 0 & 0 & 0 \\ 0 & 0 & 1 & 0 \\ 0 & 1 & 0 & 0 \\ 0 & 0 & 0 & 1\end{array}\right)\left(\begin{array}{c}\hat{S}_{-\boldsymbol{q} \uparrow} \hat{D}_{\boldsymbol{q} \uparrow} \\ \hat{S}_{-\boldsymbol{q} \uparrow} \hat{D}_{\boldsymbol{q} \downarrow} \\ \hat{S}_{-\boldsymbol{q} \downarrow} \hat{D}_{\boldsymbol{q} \uparrow} \\ \hat{S}_{-\boldsymbol{q} \downarrow} \hat{D}_{\boldsymbol{q} \downarrow}\end{array}\right)$.

Carrying out an additional reduction for a spin-singlet pairing, the interaction Hamiltonian reads

$$
\hat{\mathcal{H}}_{\mathrm{HL}-\mathrm{R}}=-\frac{J_{\mathrm{sd}}}{N} \sum_{\boldsymbol{p}, \boldsymbol{q}, \alpha} \hat{S}_{\boldsymbol{p}, \alpha}^{\dagger} \hat{D}_{-\boldsymbol{p},-\alpha}^{\dagger} \hat{D}_{-\boldsymbol{q},-\alpha} \hat{S}_{\boldsymbol{q}, \alpha}
$$

where $-\alpha$ stands for the electron spin projection opposite to $\alpha$. For comparison, we provide again the kinetic energy part of the Hamiltonian employing the same notation,

$\hat{\mathcal{H}}_{\mathrm{BH}}^{\prime}=\sum_{\boldsymbol{p}, \alpha}\left(\begin{array}{c}\hat{D}_{\boldsymbol{p}, \alpha} \\ \hat{S}_{\boldsymbol{p}, \alpha} \\ \hat{X}_{\boldsymbol{p}, \alpha} \\ \hat{Y}_{\boldsymbol{p}, \alpha}\end{array}\right)^{\dagger}\left(\begin{array}{cccc}\epsilon_{\mathrm{d}}-\mu & 0 & t_{\mathrm{pd}} s_{x} & -t_{\mathrm{pd}} s_{y} \\ 0 & \epsilon_{\mathrm{s}}-\mu & t_{\mathrm{sp}} s_{x} & t_{\mathrm{sp}} s_{y} \\ t_{\mathrm{pd}} s_{x} & t_{\mathrm{sp}} s_{x} & \epsilon_{\mathrm{p}}-\mu & -t_{\mathrm{pp}} s_{x} s_{y} \\ -t_{\mathrm{pd}} s_{y} & t_{\mathrm{sp}} s_{y} & -t_{\mathrm{pp}} s_{x} s_{y} & \epsilon_{\mathrm{p}}-\mu\end{array}\right)\left(\begin{array}{c}\hat{D}_{\boldsymbol{p}, \alpha} \\ \hat{S}_{\boldsymbol{p}, \alpha} \\ \hat{X}_{\boldsymbol{p}, \alpha} \\ \hat{Y}_{\boldsymbol{p}, \alpha}\end{array}\right)$.

Within the s-d model considered, the pairing kernel (3.4) factors into functions depending only on $\boldsymbol{p}$ or $\boldsymbol{q}$,

$$
\left(-V_{\boldsymbol{p q}}\right)=2 J_{\mathrm{sd}} S_{\boldsymbol{p}} D_{\boldsymbol{p}} S_{\boldsymbol{q}} D_{\boldsymbol{q}} \equiv 2 J_{\mathrm{sd}} \chi_{\boldsymbol{p}} \chi_{\boldsymbol{q}} .
$$

A schematic representation of the $J_{\text {sd }}$ exchange amplitude is given in figure 2 . This factorizable Markowitz-Kadanoff [60] form of the pairing kernel is a direct consequence of the local intra-atomic character of the s-d exchange in the transition ion. Substituting in equation (3.6)

$$
\Delta_{p}(T)=\Xi(T) S_{p} D_{p}=\Xi(T) \chi_{p}
$$

one obtains in a closed form, cf. reference [61], a simple BCS equation for the temperature dependence of the gap,

$$
2 J_{\mathrm{sd}}\left\langle\frac{\chi_{\boldsymbol{p}}^{2}}{2 E_{\boldsymbol{p}}} \tanh \left(\frac{E_{\boldsymbol{p}}}{2 k_{\mathrm{B}} T}\right)\right\rangle=1,
$$




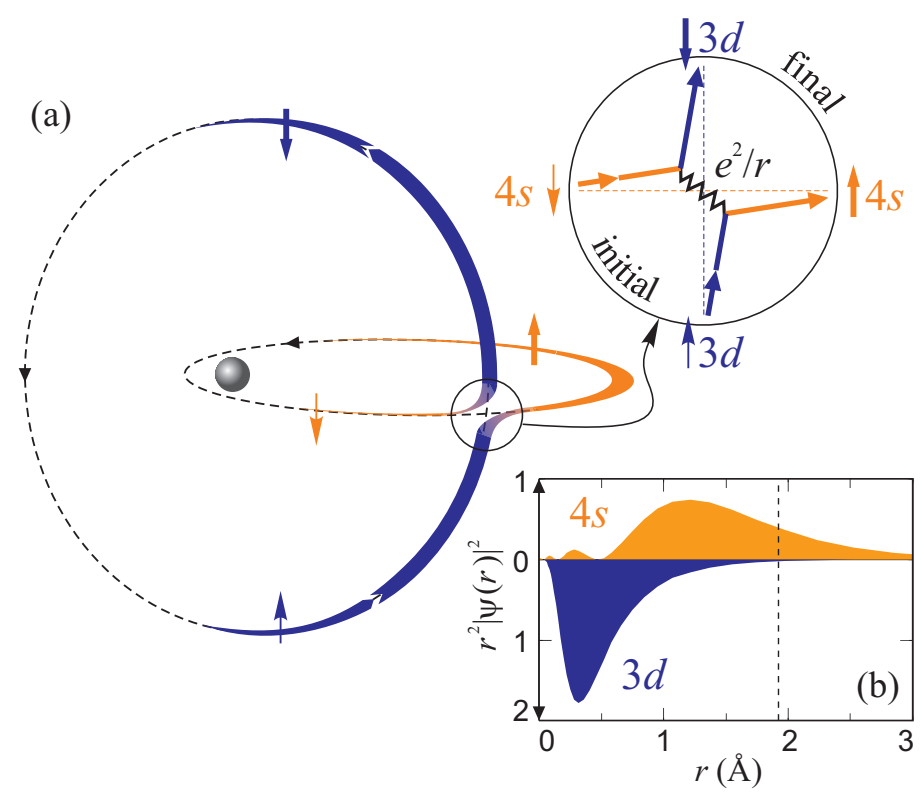

Figure 2. Pairing two-electron exchange amplitude $J_{\text {sd }}$ "hidden" in the $\mathrm{Cu}$ atom. (a) Classical Bohr-Sommerfeld representation of the s-d two-electron exchange process. The inset shows how the Coulomb scattering leads to an effective electron spin exchange. (b) Electron charge distribution for $\mathrm{Cu} 4 \mathrm{~s}$ and $\mathrm{Cu} 3 \mathrm{~d}$ orbitals: the dashed line marks the $\mathrm{Cu}-\mathrm{O}$ distance in the $\mathrm{CuO}_{2}$ plane.

where

$$
\begin{aligned}
E_{\boldsymbol{p}} & \equiv\left(\eta_{\boldsymbol{p}}^{2}+\Delta_{\boldsymbol{p}}^{2}\right)^{1 / 2}=\left[\left(\epsilon_{\boldsymbol{p}}-E_{\mathrm{F}}\right)^{2}+\left(\Xi(T) \chi_{\boldsymbol{p}}\right)^{2}\right]^{1 / 2}, \\
\left\langle f_{p}\right\rangle & =\int_{0}^{2 \pi} \int_{0}^{2 \pi} \frac{\mathrm{d} p_{x} \mathrm{~d} p_{y}}{(2 \pi)^{2}} f(\boldsymbol{p}),
\end{aligned}
$$

$E_{\mathrm{F}} \equiv \mu$. We wish to mention that separability of the order parameter (4.5) has been derived by Pokrovsky [62] in the general weak-coupling case and not only for factorizable pairing kernels.

According to (2.4) we have

$$
\begin{aligned}
\chi_{\boldsymbol{p}} \equiv S_{\boldsymbol{p}} D_{\boldsymbol{p}}=4 \varepsilon_{p} t_{\mathrm{sp}} t_{\mathrm{pd}}(x-y)\left[\varepsilon_{s} \varepsilon_{p}^{2}-4 \varepsilon_{p} t_{\mathrm{sp}}^{2}(x+y)+32 t_{\mathrm{pp}} \tau_{s p}^{2} x y\right] \\
\times\left\{\left[4 \varepsilon_{p} t_{\mathrm{sp}} t_{\mathrm{pd}}(x-y)\right]^{2}+\left[\varepsilon_{s} \varepsilon_{p}^{2}-4 \varepsilon_{p} t_{\mathrm{sp}}^{2}(x+y)+32 t_{\mathrm{pp}} \tau_{s p}^{2} x y\right]^{2}\right. \\
\left.+4 x\left[\left(\varepsilon_{s} \varepsilon_{p}-8 \tau_{s p}^{2} y\right) t_{\mathrm{pd}}\right]^{2}+4 y\left[\left(\varepsilon_{s} \varepsilon_{p}-8 \tau_{s p}^{2} x\right) t_{\mathrm{pd}}\right]^{2}\right\}^{-1} .
\end{aligned}
$$

The gap symmetry is then easily made obvious in the narrow-band approximation. Formally, it is the asymptotic behaviour of the eigenvector (2.4) for vanishing hopping integrals $t \rightarrow 0$. In this limit case [29], we have $\epsilon_{3, \boldsymbol{p}} \approx \epsilon_{\mathrm{d}}$, and

$$
\tilde{\Psi}_{3, \boldsymbol{p}}=\left(\begin{array}{c}
D_{3, \boldsymbol{p}} \\
S_{3, \boldsymbol{p}} \\
X_{3, \boldsymbol{p}} \\
Y_{3, \boldsymbol{p}}
\end{array}\right) \approx\left(\begin{array}{c}
1 \\
-\left(t_{\mathrm{sp}} t_{\mathrm{pd}} / \varepsilon_{\mathrm{s}} \varepsilon_{\mathrm{p}}\right)\left(s_{x}^{2}-s_{y}^{2}\right) \\
\left(t_{\mathrm{pd}} / \varepsilon_{\mathrm{p}}\right) s_{x} \\
\left(t_{\mathrm{pd}} / \varepsilon_{\mathrm{p}}\right) s_{y}
\end{array}\right) .
$$



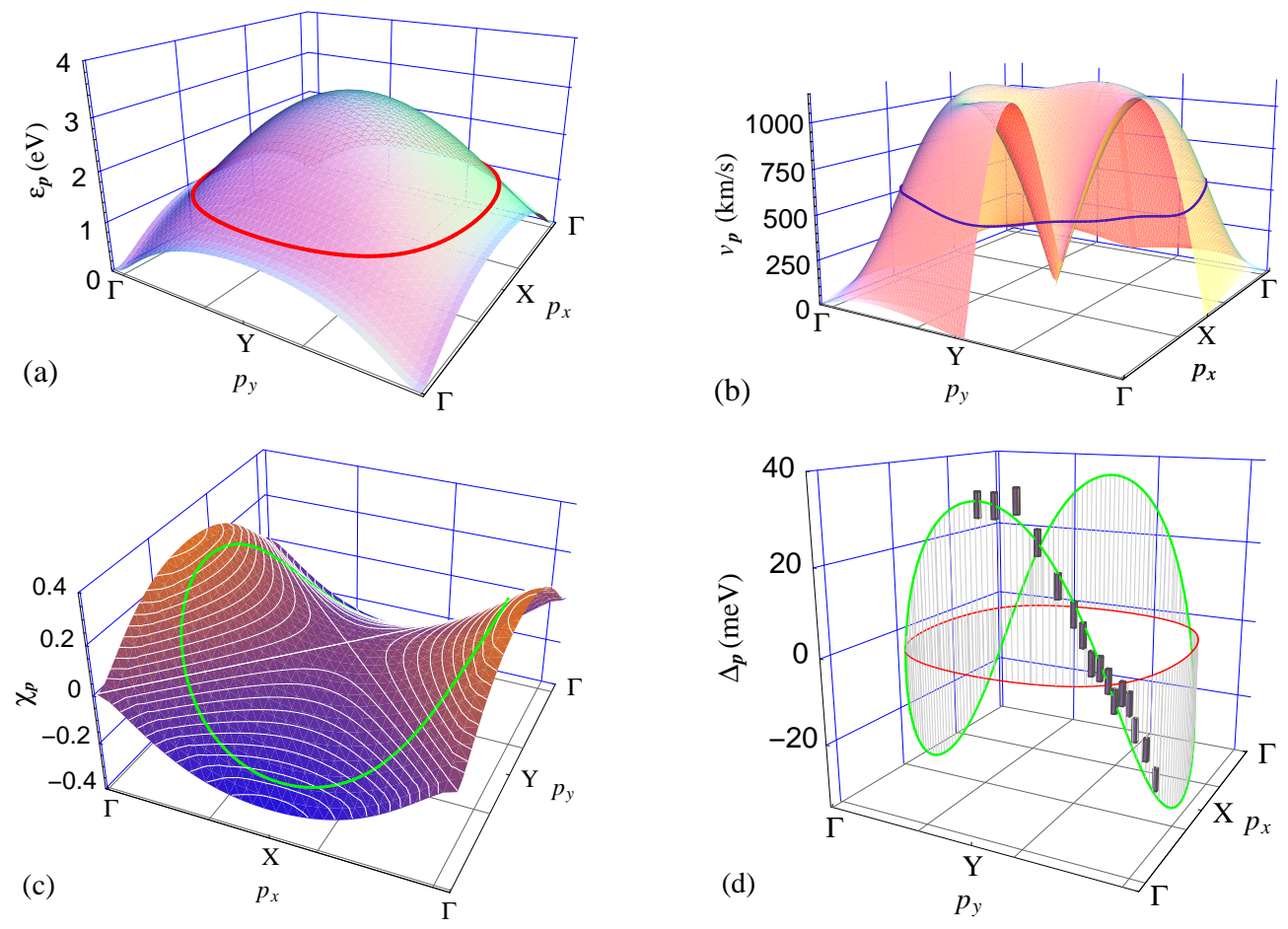

Figure 3. Electronic properties of the superconducting $\mathrm{CuO}_{2}$ plane. (a) Conduction band energy $\epsilon_{\boldsymbol{p}}$ as a function of the quasi-momentum $\boldsymbol{p}$. The red contour corresponding to the Fermi energy, $\epsilon_{\boldsymbol{p}}=E_{\mathrm{F}}$, is in excellent agreement with the ARPES data [63]. (b) Quasiparticle velocity $v_{p}$ as a function of quasimomentum. The velocity variation along the Fermi contour is less than $10 \%$. The energy parameters are fitted to be in agreement with the typical ab initio calculations [64]. The significant overestimate disappears if the bandwidth is fitted to the experimental data, but the shape is conserved. (c) Momentum dependence of the gap-anisotropy function $\chi_{p}$ within the s-d model. The functional values along the Fermi contour are indicated by a green line. (d) Superconducting gap at zero temperature $\Delta_{p}$ (green line) according to our analytical result (4.5), plotted along the Fermi contour (red line). The ARPES data [65] for $\mathrm{Bi}_{2} \mathrm{Sr}_{2} \mathrm{CaCu}_{2} \mathrm{O}_{8+\delta}$ are given as prisms with sizes corresponding to the experimental error bars. The gap function along the Fermi contour has the same qualitative behavior and symmetry as the $\mathrm{Cu} 3 \mathrm{~d}_{x^{2}-y^{2}}$ electron wave function along the circular orbit sketched in figure $2(\mathrm{a})$.

Clearly, $D_{3, \boldsymbol{p}}$ exhibits $A_{1 g}$ symmetry, while $S_{3, \boldsymbol{p}}$ has the $B_{1 g}$ symmetry. Whence the product $S_{3, \boldsymbol{p}} D_{3, \boldsymbol{p}} \propto \cos p_{x}-\cos p_{y}$ "inherits" the $B_{1 g}$ symmetry, figure 3(b), which is conserved even for realistic values of the hopping integrals, and from (4.5) it follows

$$
\Delta_{\boldsymbol{p}} \propto S_{3, \boldsymbol{p}} D_{3, \boldsymbol{p}} \approx \frac{2 t_{\mathrm{sp}} t_{\mathrm{pd}}}{\left(E_{\mathrm{F}}-\epsilon_{\mathrm{s}}\right)\left(E_{\mathrm{F}}-\epsilon_{\mathrm{p}}\right)}\left(\cos p_{x}-\cos p_{y}\right) .
$$

As can be seen in figure 3(c) this small- $t$ approximation fits the ARPES data for the gap anisotropy quite well. Similar experimental data have been previously reported, e.g., in reference [66]. Note, additionally, that close to the $(\pi, \pi)$-point, where $\left(p_{x}-\pi\right)^{2}+\left(p_{y}-\pi\right)^{2} \ll 1$, the angular dependence of the gap can be written in 
the form

$\Delta_{p} \propto \cos p_{x}-\cos p_{y} \approx\left[\left(p_{x}-\pi\right)^{2}+\left(p_{y}-\pi\right)^{2}\right] \cos 2 \phi, \quad \tan \phi=\frac{p_{y}-\pi}{p_{x}-\pi}$.

The d-type angular dependence of both the gap anisotropy and the separable pairing kernel is often postulated in phenomenological model Hamiltonians to describe high- $T_{\mathrm{c}}$ superconductivity. The previous discussion thus provides a microscopic justification based on the fundamental exchange amplitudes. For the oxygen scenario [29,67], in which the Fermi level falls in a nonbonding oxygen band, $\epsilon_{2, \boldsymbol{p}} \approx \epsilon_{\mathrm{p}}$ and $t \rightarrow 0$ [22], the gap function has different or additional nodes along the Fermi contour,

$$
\left(\begin{array}{c}
D_{2, \boldsymbol{p}} \\
S_{2, \boldsymbol{p}} \\
X_{2, \boldsymbol{p}} \\
Y_{2, \boldsymbol{p}}
\end{array}\right) \approx \frac{1}{\sqrt{s_{x}^{2}+s_{y}^{2}}}\left(\begin{array}{c}
2 t_{\mathrm{sp}}\left(t_{\mathrm{pp}} \varepsilon_{\mathrm{d}}+2 t_{\mathrm{pd}}^{2}\right)\left(\varepsilon_{\mathrm{d}} t_{\mathrm{sp}}^{2}+\varepsilon_{\mathrm{s}} t_{\mathrm{pd}}^{2}\right)^{-1} s_{x} s_{y}\left(s_{x}^{2}-s_{y}^{2}\right) \\
-s_{y} \\
s_{x}
\end{array}\right) .
$$

Here $D_{2, \boldsymbol{p}}$ and $S_{2, \boldsymbol{p}}$ exhibit $B_{2 g}$ and $A_{2 g}$ symmetries, respectively. Let us also mention that the $s$-vector components, equation (2.3), constitute the arguments of the basis functions of the symmetry representations.

Employing the analytical expression (2.8) for the constant-energy contours (CEC), one can implement an efficient numerical integration,

$$
\int_{0}^{2 \pi} \mathrm{d} p_{x} \int_{0}^{2 \pi} \mathrm{d} p_{y} f\left(\epsilon_{\boldsymbol{p}}\right)=\int_{\epsilon_{\mathrm{b}}}^{\epsilon_{\mathrm{t}}} \mathrm{d} \epsilon \oint \frac{\mathrm{d} p_{l}}{v_{\boldsymbol{p}}} f(\epsilon),
$$

where

$$
v_{\boldsymbol{p}}=\left|\frac{\partial \epsilon_{\boldsymbol{p}}}{\partial \boldsymbol{p}}\right|=\frac{\left[(\mathcal{A} y+\mathcal{B})^{2} x(1-x)+(\mathcal{A} x+\mathcal{B})^{2} y(1-y)\right]^{1 / 2}}{\left|\mathcal{A}^{\prime} x y+\mathcal{B}^{\prime}(x+y)+\mathcal{C}^{\prime}\right|},
$$

with $\mathcal{A}^{\prime}, \mathcal{B}^{\prime}$ and $\mathcal{C}^{\prime}$ being the energy derivatives of the polynomials (2.9),

$$
\begin{aligned}
& \mathcal{A}^{\prime}(\epsilon)=16\left[2 t_{\mathrm{sp}}^{2} t_{\mathrm{pp}}-2 t_{\mathrm{pd}}^{2} t_{\mathrm{pp}}-t_{\mathrm{pp}}^{2}\left(\varepsilon_{\mathrm{d}}+\varepsilon_{\mathrm{s}}\right)\right], \\
& \mathcal{B}^{\prime}(\epsilon)=-4\left(t_{\mathrm{sp}}^{2} \varepsilon_{\mathrm{d}}+t_{\mathrm{pd}}^{2} \varepsilon_{\mathrm{s}}\right)-4 \varepsilon_{\mathrm{p}}\left(t_{\mathrm{sp}}^{2}+t_{\mathrm{pd}}^{2}\right), \\
& \mathcal{C}^{\prime}(\epsilon)=\varepsilon_{\mathrm{s}} \varepsilon_{\mathrm{p}}^{2}+\varepsilon_{\mathrm{d}} \varepsilon_{\mathrm{p}}^{2}+2 \varepsilon_{\mathrm{d}} \varepsilon_{\mathrm{s}} \varepsilon_{\mathrm{p}} .
\end{aligned}
$$

Using these functions, the band spectrum, see (2.8), can be obtained by Newton iterations

$$
\epsilon_{\boldsymbol{p}}^{[i]}=\epsilon_{\boldsymbol{p}}^{[i-1]}-\frac{\mathcal{A} x y+\mathcal{B}(x+y)+\mathcal{C}}{\mathcal{A}^{\prime} x y+\mathcal{B}^{\prime}(x+y)+\mathcal{C}^{\prime}}
$$

with initial approximation for the conduction band $\epsilon_{3, \boldsymbol{p}}^{[0]}=\varepsilon_{\mathrm{d}}$.

The charge carrier velocity is $v_{\boldsymbol{p}} a_{0} / \hbar, a_{0}$ is the lattice constant, $p_{l}$ the dimensionless momentum component along the CEC, and $\epsilon_{\mathrm{b}}$ and $\epsilon_{\mathrm{t}}$ are the bottom and the top of the conduction band, respectively, $\epsilon_{\mathrm{b}} \leq \epsilon_{\boldsymbol{p}} \leq \epsilon_{\mathrm{t}}$. The canonic equation for the CEC (2.8),

$$
\mathcal{A} \cos p_{x} \cos p_{y}-(\mathcal{A}+2 \mathcal{B})\left(\cos p_{x}+\cos p_{y}\right)+\mathcal{A}+4 \mathcal{C}=0,
$$

can be cast in an explicit form

$$
p_{y, 1}\left(p_{x}\right)=2 \arcsin \sqrt{-\frac{\mathcal{B} x+\mathcal{C}}{\mathcal{A} x+\mathcal{B}}}, \quad p_{y, 2}\left(p_{x}\right)=2 \pi-p_{y, 1}\left(p_{x}\right),
$$


and for the length element $\mathrm{d} p_{l}$ we obtain

$$
\mathrm{d} p_{l}=\sqrt{1+\left(\frac{\mathrm{d} p_{y}}{\mathrm{~d} p_{x}}\right)^{2}} \mathrm{~d} p_{x}, \quad\left(\frac{\mathrm{d} p_{y}}{\mathrm{~d} p_{x}}\right)^{2}=\frac{x(1-x)}{y(1-y)}\left(\frac{\mathcal{A} y+\mathcal{B}}{\mathcal{A} x+\mathcal{B}}\right)^{2} .
$$

The contour integration along the hole pocket $\varepsilon_{\boldsymbol{p}}=$ const centered at the $(\pi, \pi)$ point needs to be performed only over one eight of the CEC

$$
\oint \mathrm{d} p_{l} f\left(p_{x}, p_{y}\right)=8 \int_{p_{d}}^{\pi} f\left(p_{x}, p_{y}\left(p_{x}\right)\right)\left(\frac{\mathrm{d} p_{d}\left(p_{x}\right)}{\mathrm{d} p_{x}}\right) \mathrm{d} p_{x},
$$

where

$$
x_{d}=\sin ^{2}\left(\frac{p_{d}}{2}\right), \quad \mathcal{A} x_{d}^{2}+2 \mathcal{B} x_{d}+\mathcal{C}=0 .
$$

\section{Antiferromagnetic character of $J_{\mathrm{sd}}$}

Let us address now the atomic physics underlying the s-d pairing mechanism. Within the framework of the Hartree-Fock (HF) theory the exchange energy is given [68] as an integral of the $\mathrm{Cu} 4 \mathrm{~s}$ and $\mathrm{Cu} 3 \mathrm{~d}_{x^{2}-y^{2}}$ atomic wave functions,

$$
-J_{\mathrm{sd}}^{(\mathrm{HF})}=\iint \psi_{\mathrm{s}}^{*}\left(\boldsymbol{r}_{1}\right) \psi_{\mathrm{d}}^{*}\left(\boldsymbol{r}_{2}\right) \frac{e^{2}}{\left|\boldsymbol{r}_{1}-\boldsymbol{r}_{2}\right|} \psi_{\mathrm{d}}\left(\boldsymbol{r}_{1}\right) \psi_{\mathrm{s}}\left(\boldsymbol{r}_{2}\right) \mathrm{d} \boldsymbol{r}_{1} \mathrm{~d} \boldsymbol{r}_{2},
$$

and its sign corresponds to repulsion and depairing for singlet Cooper pairs. Thus, one can formulate the following conceptual problem, emerging in fundamental physics:

(i) is it possible, as in the case of a covalent bond, for two-electron correlations to trigger a change of the sign of the exchange amplitude?

(ii) how can one adapt the Heitler-London idea to a transition ion perturbed by ligands?

There is no doubt that the solution to this problem will illuminate other problems in the physics of magnetism as well. In brief, the enigma can be stated as to whether the Heitler-London approximation for the exchange energy may result in $J_{\mathrm{sd}}>0$, cf. reference [68]. Let us recall that already in 1962 Herring [45] was advocating that "antiferromagnetic $J_{i j}$ 's should be the rule, ferromagnetic $J_{i j}$ 's the exception". For the present, we can adopt the s-d model as a convenient microscopic phenomenology of superconductivity in the $\mathrm{CuO}_{2}$ plane. On the other hand, the exchange amplitude $J_{\text {sd }}$ is an important ingredient in the physics of magnetism as well.

Physics of magnetism certainly displays lots of subtleties, but for a qualitative comparison let us trace the "operation" of the s-d exchange amplitude $J_{\text {sd }}$ in the case of the simplest model for a ferromagnetic metal. While for the $\mathrm{CuO}_{2}$ plane the s-band is empty, for transition metals it is the widest conduction band. The width of the d-band is significantly smaller and thus, making a caricature of the ferromagnetic metals, we completely neglect the width of the d-band. In this "heavy Fermion" approximation the d-electrons are considered as localized, and without significant energy loss they can be completely spin polarized, $\left\langle\hat{n}_{\mathrm{d} \uparrow}\right\rangle \approx 1,\left\langle\hat{S}_{\mathrm{d}, z}\right\rangle \approx \frac{1}{2}>0$. In this case the self-consistent approximation applied to (2.17) gives

$$
2 \hat{\boldsymbol{S}}_{\mathrm{d}} \cdot \hat{\boldsymbol{S}}_{\mathrm{s}} \approx \hat{S}_{\mathrm{d}, z}\left(\hat{n}_{\mathrm{s} \uparrow}-\hat{n}_{\mathrm{s} \downarrow}\right) \approx\left\langle\hat{S}_{\mathrm{d}, z}\right\rangle\left(n_{\mathrm{s} \uparrow}-n_{\mathrm{s} \downarrow}\right) .
$$

Here $n_{\mathrm{s} \alpha} \equiv\left\langle\hat{n}_{\mathrm{s} \alpha}\right\rangle$ denotes the average number of s-electrons per atom with spin projection $\alpha$. In order to calculate these variables one has to take into account the 
different filling of the s-bands with different polarizations, and sum over the quasimomenta. Finally, the exchange energy per atom reads

$$
E_{\mathrm{X}}=-\frac{1}{2} J_{\mathrm{sd}}\left(n_{\mathrm{s} \downarrow}-n_{\mathrm{s} \uparrow}\right)<0 .
$$

In the $\mathrm{CuO}_{2}$ plane, positive values of the $J_{\mathrm{sd}}$ parameter lead to singlet superconductivity. For ferromagnetic metals, positive values of $J_{\mathrm{sd}}$ correspond to polarization of the s-band opposite to d-state polarization, $n_{\mathrm{s} \downarrow}-n_{\mathrm{s} \uparrow}>0$. Thereby ferromagnetism could be brought about by an exchange amplitude with a sign corresponding to antiparallel spin polarization of s- and d-orbitals, cf. figure 4-15 of reference [52]. Thus the same sign of the s-d exchange amplitude $J_{\mathrm{sd}}$ can be at the origin of ferromagnetism, e.g., in $\mathrm{Fe}$ and $\mathrm{Ni}$, and superconductivity in the $\mathrm{CuO}_{2}$ plane. This is perhaps the simplest scenario for cuprate superconductivity based on the two-electron exchange processes.

According to a naive interpretation of Hund's rule the Kondo effect should not exist. In the epoch-making paper [69] on the resistance minimum in dilute magnetic alloys Kondo concluded that in the s-d exchange model, due to Zener [20], Kasuya and Yosida [70], the sign of the direct exchange amplitude $J_{\text {sd }}$ must be antiferromagnetic. And vice versa, the minimum disappears if $J_{\text {sd }}$ is ferromagnetic. Such minimum exists for many magnetic metals and alloys and is another hint in favor of Herring's argument [45] mentioned earlier. In his analysis Kondo speculates that $J_{\mathrm{sd}}$ is a parameter whose sign and magnitude have to be determined so as to fit the experiment, and concluded that antiferromagnetic values of the order of $\mathrm{eV}$ are quite reasonable. For a review on the Kondo problem we refer the reader to reference [71].

On the other hand every textbook on atomic physics tells us that parallel electron spins and an antisymmetric wave function minimize the electrostatic energy. Put differently, the tendency toward ferromagnetism in Hund's rule is of electrostatic origin. As Kondo has pointed out [69], the problem is to find the origin of an antiferromagnetic $J_{\mathrm{sd}}$, or how to overcome the strong electrostatic repulsion. It is very plausible that it is not a single driving force, but instead one has to take into account several interfering electron scattering amplitudes.

\subsection{Intra-atomic correlations}

The self-consistent approximation has been known in celestial mechanics for ages. Accordingly, the motion of a planet is averaged over its orbit. One has to calculate then the potential created by this orbital-averaged motion and perform a sum over all particles. Where does this scheme fail? It fails in the case of a resonance when the periods for some planets are commensurate or just equal. This is nothing but the case of a transition ion for which the energies and classical periods are very close. Then the resonant repetitive electron scattering, symbolically presented in figure 2 , leads to strong electron correlations like in the double Rydberg states of atoms [35]. For double Rydberg states in He it is necessary to solve a two-electron quantum problem but for other atoms we have to take into account the influence of the other electrons in some self-consistent approximation, the local density approximation (LDA), for example. For two 4s electrons the two-electron correlations are so strong that they have to be taken into account from the very beginning [35]. There are no doubts that the two-electron correlations between $4 \mathrm{~s}$ and $3 \mathrm{~d}$ electrons having almost equal energies cannot be neglected. In other words Hartree-Fock theory cannot be used directly. Hence, the Bohr picture is not merely a historical remark but rather an indispensable 
ingredient of the contemporary physics of magnetism: two-electron correlations can be important even in a single atom. We thus conclude that the two-electron correlations may overrule Hund's rule for the local s-d exchange. Note also that the single-particle orbitals (accessible, e.g., from DFT [48], HF and $X_{\alpha}$ methods, etc.) only form an adequate basis for a subsequent account of electronic correlations. A first step in this direction will be $a b$ initio calculation of the $J_{\mathrm{sd}}$.

\subsection{Indirect $s$-d exchange}

The antiferromagnetism of the insulating phase of the undoped cuprates is mediated by the Bloch-Kramers-Anderson indirect exchange [72] between 3d electrons of nearestneighbour $\mathrm{Cu}$ ions via $\mathrm{O} 2 \mathrm{p}$ electrons. It is unlikely that the numerical value of this $J_{\mathrm{dd}}$ exchange integral be dramatically changed in the metallic phase obtained by hole doping. In the metallic phase, however, the same indirect exchange mechanism will operate between $3 \mathrm{~d}$ and $4 \mathrm{~s}$ electrons at the same $\mathrm{Cu}$ atom via the $2 \mathrm{p}$ electrons of the O ligands. For illustration, let us compare the indirect s-d exchange amplitude $J_{\text {sd }}^{\text {(ind) }}$ with $J_{\mathrm{dd}}$. There are three important factors: (i) Every $\mathrm{Cu}$ ion has four $\mathrm{O}$ ligands, figure 1 (a). (ii) The hopping amplitude between $4 \mathrm{~s}$ and $2 \mathrm{p}$ orbitals is bigger than the $3 \mathrm{~d}-2 \mathrm{p}$ transfer. (iii) The $\mathrm{Cu}$ on-site Coulomb repulsion between $4 \mathrm{~s}$ and $3 \mathrm{~d}$ electrons $U_{\text {sd }}$ is much smaller than the $3 \mathrm{~d}-3 \mathrm{~d}$ Hubbard repulsion $U_{\mathrm{dd}}$. Taking into account these factors one can expect that $J_{\mathrm{sd}}^{(\text {ind) }}$ is an order of magnitude bigger than $J_{\mathrm{dd}}$ :

$$
J_{\text {sd }}^{\text {(ind) }} \simeq 4\left(\frac{t_{\mathrm{sp}}}{t_{\mathrm{pd}}}\right)^{2} \frac{U_{\mathrm{dd}}}{U_{\mathrm{sd}}} J_{\mathrm{dd}}
$$

The relatively small $J_{\mathrm{dd}}$ ensures Néel temperatures $T_{\mathrm{N}}$ of the order of room temperature. Hence we can conclude that the indirect exchange can contribute significantly to the total $J_{\text {sd }}$ amplitude responsible for the pairing. However, only very detailed first-principles calculations can clarify the relative contributions of the direct and the indirect s-d exchange.

\subsection{Effect of mixing wave functions}

In an early paper [73], by analyzing the $g$-shift and the anomalous Hall effect in Gd metal, Kondo showed that an antiferromagnetic $J_{\text {sd }}$ can result from the effect of mixing the wave functions of conduction and d electrons. We believe that this property is preserved if the d electrons also form a conduction band, or even in the case of a single s-p-d hybridized band. We should note that Kondo's argumentation for the need of a $J_{\text {sd }}$ with an antiferromagnetic sign in the Kondo effect is related to Anderson's consideration of localized magnetic states in metals [74]. In the latter schematized model, based on the works of Friedel [75], Anderson shows that "any $g$-shift caused by free-electron polarization will tend to have antiferromagnetic sign."

As it was expected by various investigators the later numerical calculations confirmed that the striking features of negative hyperfine field with large amplitude comes mainly from the contact contribution of the core electrons [76-78]. The antipolarization between the s- and d-electrons in transition metal compounds is also well observed by Mössbauer spectroscopy, however the contribution of the core selectrons and conduction band cannot be experimentally resolved. For the pairing, the amplitude of the s-d Kondo scattering is essential because in some terminology the $\mathrm{CuO}_{2}$ plane can be considered to be a single-band Kondo lattice, cf. reference [79]. 
Given the above diversity of channels for s-d exchange it is not surprising that an adequate first-principles scheme to calculate $J_{\text {sd }}$ is still sought. Furthermore, $J_{\text {sd }}$ is involved in the theory of magnetism in an entangled way precluding so far a direct relation between $a b$ initio calculations and formulae fitting the experiment [80].

\subsection{Cooper and Kondo singlet formation}

Although Kondo [69] does cite Zener's paper [20], in many publications in the field of magnetism the s-d interaction is referred to as Kondo interaction. Often this term is used in a broader sense causing eventually terminological misunderstanding. Here we cite some works which could be related to our theory even though the relation may not be direct or immediately apparent. Analyzing the possible "interplay of Cooper and Kondo singlet formations in high- $T_{c}$ cuprates" Sekitani et al [81] point out that "In the $20^{\text {th }}$ century, two significant many-body phenomena due to spin singlet formation

were discovered in the field of solid state physics: superconductivity and the Kondo effect". These authors believe that the pseudogap in the normal state corresponds to the dissociation energy of the Kondo bound states and that superconductivity and the Kondo effect are competing in $\mathrm{La}_{1.85} \mathrm{Sr}_{0.15} \mathrm{CuO}_{4}$. They speculate that the interplay between superconductivity and the Kondo effect has not be considered seriously for high- $T_{\mathrm{c}}$ cuprates (further references on the Kondo effect in cuprate superconductors can be found, e.g., in [82]). It would be premature for us to comment on these ideas; we note however that within this terminology our theory could be considered as a Kondo interaction mechanism for pairing in high- $T_{\mathrm{c}}$ cuprates.

It is unclear from microscopic point of view if the same "Kondo interaction" amplitude $J_{\mathrm{sd}}$ is responsible for the empirical Kadowaki-Woods ratio [83] but the location of $\mathrm{La}_{1.7} \mathrm{Sr}_{0.3} \mathrm{CuO}_{4}$ on the $A$ (the coefficient of the $T^{2}$ resistivity) versus $\gamma_{0}$ (the electronic specific-heat coefficient) plot, cf. figure 4 in [84], is a serious hint that $J_{\text {sd }}$ in the $\mathrm{CuO}_{2}$ plane is one of the largest exchange amplitudes in solid state physics, comparable with the uranium heavy-fermion compounds and $\mathrm{SrVO}_{3}$. In this sense our theory requires a large, yet acceptable $J_{\text {sd }}$ value, putting the cuprates among the most interesting materials with considerable exchange interaction.

\section{Dogmatics, Discussion, Conclusions and Perspectives}

In a review on the history of studies of superconductivity and the prospects for further research in the field Ginzburg [2] conditionally divided the history into several periods:

(i) The "Day Before Yesterday" (1911-1941). This period starts with the discovery of superconductivity in Leiden by Gilles Holst and Heike Kamerlingh Onnes [2, 85].

(ii) "Yesterday" (1942-1986). This period embraces the appearance of the $\Psi-\Delta$ theories and the first significant technical applications.

(iii) "Today" (1987-?). This epoch emerged with the discovery of the high- $T_{\mathrm{c}}$ cuprates [1].

(iv) "Tomorrow" (?). The final landmark of "Today" must be some event.

Long ago, in the "Day Before Yesterday" high- $T_{\mathrm{c}}$ superconductivity was known as a "blue dream" of physicists. Considerable theoretical efforts were applied "Yesterday", attempting to predict possible realizations of this phenomenon [86]. At that time the problem of high- $T_{\mathrm{c}}$ superconductivity was "one of the most interesting and attractive 
problems from the purely scientific point of view" [86]. Intriguingly, the special role of layered metallic systems and almost two-dimensional superconductivity [87] was mentioned already in this epoch, and a big variety of mechanisms of superconductivity were considered including the s-d exchange $[88,89]$. This exchange process was well known in the physics of magnetism since the dawn of quantum mechanics. Thus it is not surprising that the first work on the s-d pairing mechanism, by Akhiezer and Pomeranchuk [88], was accomplished about a year after the celebrated BCS paper [3]. These pioneering works, however, "have been ignored thus far" [90].

After Bednorz and Müller's work [1] the problem of high- $T_{\mathrm{c}}$ superconductivity soon came into fashion. "After experiencing the 'smell of roast meat', yesterday skeptics or even critics can become zealous advocates of a new direction of endeavor. But this is another story-more in the realm of psychology and sociology than scientific and technical activity" [91]. All models of high- $T_{\mathrm{c}}$ superconductivity were revisited in great detail in the uncountable number of papers that have appeared in the epoch "Today".

\subsection{Aesthetics and frustrations of the central dogmas}

The common trends of some new theoretical models for cuprate superconductivity were systematized by Anderson [92] in six dogmas. We find it very instructive to compare our theory of high- $T_{\mathrm{c}}$ superconductivity with these dogmas.

"Dogma I: All the relevant carriers of both spin and electricity reside in the $\mathrm{CuO}_{2}$ plane and derive from the hybridized $\mathrm{O} 2 p-C u 3 d_{x^{2}-y^{2}}$ orbital which dominates the binding in these compounds. ... in summary look at the planes only (a great and welcome simplification.)"

The key ingredient of our pairing theory is the four-fermion s-d interaction between the $\mathrm{Cu} 4 \mathrm{~s}$ and $\mathrm{Cu} 3 \mathrm{~d}_{x^{2}-y^{2}}$ orbitals. If we cut the $\mathrm{Cu} 4 \mathrm{~s}$ orbital off from the Hilbert space of the $\mathrm{CuO}_{2}$ plane such a pairing interaction cannot exist. Although $\mathrm{Cu} 4 \mathrm{~s}$ is an empty band, it is an important component of the theory of high- $T_{\mathrm{c}}$ superconductivity. The $\mathrm{O} 2 \mathrm{p}$ orbitals are the intermediaries between the $\mathrm{Cu} 4 \mathrm{~s}$ and $\mathrm{Cu} 3 \mathrm{~d}_{x^{2}-y^{2}}$ orbitals that create the necessary s-d hybridization of the conduction $\mathrm{Cu} 3 \mathrm{~d}_{x^{2}-y^{2}}$ band.

"Dogma II: Magnetism and high- $T_{\mathrm{c}}$ superconductivity are closely related, in a very specific sense: i.e., the electrons which exhibit magnetism are the same as the charge carriers. ... We must solve the old problem of doping of a single Mott-Hubbard band before we can begin the problem of high-T $T_{\mathrm{c}}$."

The incommensurate spin-density waves (SDW) observed in the superconducting phase of $\mathrm{La}_{2} \mathrm{CuO}_{4.11}$ and $\mathrm{La}_{1.88} \mathrm{Sr}_{0.12} \mathrm{O}_{4}$ by neutron scattering [93] and muon spin relaxation [94], respectively, demonstrated that antiferromagnetism of the $\mathrm{Cu}$ site is innocuous for superconductivity in the cuprates. These antiferromagnetic correlations are not depairing and do not change significantly $T_{\mathrm{c}}$ and the electronic structure of $\mathrm{CuO}_{2}$ plane. The observed correspondence between the magnetic and the superconducting order parameters is an additional hint that both phenomena have a common origin [95]; see also the detailed theoretical works [96]. Nevertheless the coexistence of SDW and superconductivity with a common critical temperature cannot be clearly observed in every high- $T_{\mathrm{c}}$ cuprate. As a result superconductivity can be considered, at least in first approximation, separately from a possible antiferromagnetism as is done in the present paper. In $\mathrm{Cr}$ metal the amplitude of the SDW shows also a BCS-like temperature dependence [97] and the SDW-theory is 
based on the conventional theory of metals based in turn on the Landau Fermi-liquid theory. We consider the quasiparticle picture as a reliable starting point for the theory of high- $T_{\mathrm{c}}$ cuprates as well.

"Dogma III: The dominant interactions are repulsive and their energy scales are all large. ... Restrict your attention to a single band, repulsive (not too big) U Hubbard Model."

Indeed, the dominant interactions are repulsive - "Nobody has abrogated the Coulomb law", as Landau used to emphasize [2]. However, something subtle occurs when the atomic orbitals are analyzed. The strong electron repulsion leads to HeitlerLondon type correlations: two electrons cannot occupy simultaneously the same orbital, not even if they have opposite spins. The exchange of electrons between two orbitals decreases the electron kinetic energy and thereby the total energy of the whole system. In molecular physics, according to the Hellmann-Feynman theorem such a decrease in energy drives an inter-atomic attraction for large inter-atomic distances. Thus, the valence attraction is the final result of the dominant Coulomb repulsion between electrons. In this way the Heitler-London-type exchange between itinerant electrons gives rise to electron-electron attraction and conventional Cooper pairing. The s-d exchange, "residing" in the $\mathrm{Cu}$ atom, can be considered as an "intra-atomicvalence bond" - an attraction-sign scattering amplitude due to the Coulomb repulsion between the correlated electrons. The s-d exchange in the transition ions is one of the most intensive exchange processes in solid state physics. Such a high-frequency process is described by the exchange amplitude $J_{\text {sd }}$ in the lattice models for the electronic structure and its sign is determined by the inter-electronic Coulomb repulsion. The Heitler-London interaction is a result of strong electron repulsion and survives even for infinite Hubbard $U$. This interaction is lost when starting with the infinite- $U$ Hubbard model, however. Thus, not a single-band Hubbard model but a single-band s-d model with antiferromagnetic exchange amplitude is the adequate starting point for a realistic treatment of $\mathrm{CuO}_{2}$ superconductivity.

"Dogma IV: The 'normal' metal above $T_{\mathrm{c}}$... is not a Fermi liquid. . but retains a Fermi surface satisfying Luttinger's theorem at least in the highest- $T_{\mathrm{c}}$ materials. We call this a Luttinger Liquid."

Very recently, the crucial experiment has finally been conducted. After 15 years of intensive investigations of the cuprates it is now experimentally established [99] that the overdoped cuprates obey the 150-years-old Wiedemann-Franz law within a remarkable $1 \%$ accuracy. After this experimental clarification the theoretical comprehension will hardly keep us waiting long. This experiment has also solved the old problem of the nature of charge carriers created by doping of a single MottHubbard band, cf. Dogma II. Now we know that charge carriers of the normal state are standard Landau quasi-particles [100] for which we have conventional Cooper pairing in the superconducting phase. "Holons", "spinons" and spin-charge separation are unlikely to occur and behave so as to emulate the properties of the ideal Fermi gas. As a function of the hole doping per $\mathrm{Cu}$ atom, $\tilde{p}$, the critical temperature is a smooth parabola [98],

$$
T_{\mathrm{c}} / T_{\mathrm{c}}^{\max } \approx 1-82.6(\tilde{p}-0.16)^{2} .
$$

Thus, it is improbable that the nature of the carriers and pairing mechanism can be dramatically changed in the optimal and underdoped regimes although a number of new and interesting phenomena complicate the physics of the underdoped cuprates.

In short, in our opinion the experimental validation of the Wiedemann-Franz 
law in overdoped cuprates [99] is a triumph of the Landau [100] and Migdal concept of Fermi quasi-particles (and Landau spirit of trivialism in general) and provides a refutation of the spin-charge separation in cuprates [101]. Hence, the problem of deriving the Wiedemann-Franz law for strongly correlated electrons in the $\mathrm{CuO}_{2}$ plane has just been set in the agenda. According to the Fermi liquid theory [102] interactions between the particles create an effective self-consistent Hamiltonian. As Kadanoff [103] has pointed out, this idea was much developed by Landau [104] and Anderson [105]. Unfortunately, for high- $T_{\mathrm{c}}$ cuprates a link is still missing between the Landau quasiparticle concept and the one due to Slater that even scattering matrix elements can be calculated from first principles.

"Dogma V: Nonetheless, enough directions have been probed to indicate strongly that this odd-even splitting of $\mathrm{CuO}_{2}$ planar states doesn't exist. ... The impact of Dogma $V$, then, is that the two-dimensional state has separation of charge and spin into excitations which are meaningful only within their two-dimensional substrate; to hop coherently as an electron to another plane is not possible, since the electron is a composite object, not an elementary excitation."

Within the single-particle approximation (section 2) the bilayer band splitting is readily obtained from (2.8) and (4.19) by the replacements

$$
\epsilon_{i} \rightarrow \epsilon_{i} \pm t_{\perp, i i}, \quad i=\mathrm{s}, \mathrm{p}, \mathrm{d},
$$

where $t_{\perp, i i}$ is the hopping amplitude between the $i$ th orbitals in the adjacent $\mathrm{CuO}_{2}$ planes. In other words, the two constant energy curves due to the bilayer splitting are described by the same equation (2.8). Since it is plausible that $t_{\perp, \mathrm{ss}}$ dominates, from (4.10) one finds

$$
\Delta E_{\text {bilayer }} \approx 2 t_{\perp, \mathrm{ss}}\left|S_{3, \boldsymbol{p}}\right|^{2} \approx 22 \mathrm{meV}\left(\cos p_{x}-\cos p_{y}\right)^{2},
$$

in agreement with references $[64,106]$. The numerical value of $22 \mathrm{meV}$ has been reported for heavily overdoped $\mathrm{Bi}_{2} \mathrm{Sr}_{2} \mathrm{CaCu}_{2} \mathrm{O}_{8+\delta}$ (BSCCO) [106]. This experiment, crucial for Dogma $V$, cf. reference [107], is another piece of evidence in favor of the conventional behaviour of the electron excitations in the $\left(\mathrm{CuO}_{2}\right)_{2}$ slab. Since $\Delta E_{\text {bilayer }}$ is relatively small in comparison with the width of the conduction band, it is another hint that even for bilayer superconductors like BSCCO and $\mathrm{YBa}_{2} \mathrm{Cu}_{3} \mathrm{O}_{7-\delta}(\mathrm{YBCO})$ the analysis of a single $\mathrm{CuO}_{2}$ plane is an acceptable initial approximation. Similarly, for fitting the three-dimensional Fermi surface of $\mathrm{Tl}_{2} \mathrm{Ba}_{2} \mathrm{CuO}_{6+\delta}$ determined by angle magnetoresistance oscillations [108] one can start with the simplest possible tightbinding approximation

$$
\epsilon_{i} \rightarrow \epsilon_{i}+t_{\perp, i i} \cos p_{z}, \quad i=\mathrm{s}, \mathrm{p}, \mathrm{d} .
$$

"Dogma VI: Interlayer hopping together with the "confinement" of Dogma V is either the mechanism of or at least a major contributor to superconducting condensation energy."

The interlayer hopping which is understood as a single-electron process definitely cannot be considered as a two-electron pairing interaction creating the condensation energy. It is only one of the details when one concentrates on the material-specific effects in high- $T_{\mathrm{c}}$ superconductors. The inter-slab hopping between double $\left(\mathrm{CuO}_{2}\right)_{2}$ layers is a coherent Josephson tunnelling responsible for the long-living plasma oscillations with frequency $\omega_{\mathrm{pl}}<\Delta$. These plasma oscillations along with far infrared transparency of the superconducting phase were theoretically predicted [109] for $\mathrm{BSCCO}$ - one of the few predictions made for high- $T_{\mathrm{c}}$ cuprates, cf. the postdiction [110]. After the experimental observation [111], the plasma resonances associated 
with the Cooper-pair motion soon turned into a broad research field [112]. Subgap plasmons were predicted [113] for conventional superconducting thin films as well, and shortly after experimentally confirmed [114] for thin Al films on $\mathrm{SrTiO}_{3}$ substrate. The relatively lagged development of the physics of this effect was partially due to the false neglect of the longitudinal current response in the classical works on microscopic theory. Concluding, let us note that the London penetration depth $\lambda$ can be considered as the Compton wave length of the Higgs boson of mass $m_{\mathrm{H}} c^{2}=\hbar \omega_{\mathrm{pl}}$, but the overall contribution of the interlayer hopping to the condensation energy is negligible.

\subsection{Discussion}

The band structure of the $\mathrm{CuO}_{2}$ plane is now believed to be understood. However, after 15 years of development a mismatch of a factor of two or three between the $a b$ initio and the experimental spectroscopic estimates for the single-electron hopping amplitudes $t$, or the bandwidth, tends to be interpreted rather as a stateof-the-art "coincidence". The Heitler-London approach is well-known in quantum chemistry $[115,116]$, and has been successfully used for a long time in the physics of magnetism [117]. We hope that realistic first-principles calculations aiming at the exchange integrals $J$ of the $\mathrm{CuO}_{2}$ plane can be easily carried out. Should they validate the correct (antiferromagnetic) sign and the correct order of magnitude of $J_{\text {sd }}$, we can consider the theory of high- $T_{\mathrm{c}}$ superconductivity established. We stress that the twoelectron exchange, analyzed here, is completely different from the double exchange considered in reference [118].

In order to compare the derived results with the experiment, it is necessary that the tight-binding conduction band energy be fitted to the available ARPES data. In doing so a few parameters have to be properly taken into account: the Fermi energy $E_{\mathrm{F}}$, as determined from the total area of the hole Fermi contour, the difference between the Fermi energy and the Van Hove singularity, $E_{\mathrm{F}}-\epsilon(\pi, 0)$, the difference between the Van Hove singular point and the bottom of the conduction band at the $\Gamma$ point, $\epsilon(\pi, 0)-\epsilon(0,0)$. The fit may further allow taking into account a possible realization of the Abrikosov-Falkovsky scenario, cf. reference [29]. According to the latter, for $\varepsilon_{\mathrm{d}}<\varepsilon_{\mathrm{p}}<\varepsilon_{\mathrm{s}}$ and sufficiently small $t_{\mathrm{pd}}$, the conduction band can be the narrow (nonbonding) oxygen band having a perfect (within the framework of the four-band model) extended Van Hove singularity. If the superconducting gap has a $B_{1 g}$-type symmetry, its maximum value along the Fermi contour, $\Delta_{\max }=\max \left|\Delta_{p}(T=0)\right|$, determines the $J_{\text {sd }}$ exchange integral in the s-d model. Thus, the temperature dependence of the gap, described by the function $\Xi(T)$, and the overall thermodynamic behaviour and low frequency electrodynamic response will be determined without free fitting parameters.

The fit to the extended Van Hove singularity as observed, e.g., in reference [119] also points to a relatively low-lying $\mathrm{Cu} 4 \mathrm{~s}$ level and one needs to consider the minimum value of $\epsilon_{\mathrm{s}}$. Although the $4 \mathrm{~s}$ band is completely empty $(\mathrm{Cu} 4 \mathrm{~s}$ level is above the Fermi level), a very close location is not "harmless" and would necessarily lead to some prediction for the optical behaviour. With some risk of opening the Pandora's box, we should mention that the lowest position of the $\mathrm{Cu} 4 \mathrm{~s}$ level is determined by the mid-infrared (MIR) response. According to this possible interpretation, the broadly discussed maximum of the absorption in the MIR range is due to $3 \mathrm{~d}-4 \mathrm{~s}$ interband transition: one electron in the conduction band is excited by the light to the empty $\mathrm{Cu} 4 \mathrm{~s}$ band. It seems that, up to now, there is no natural explanation of this MIR 
optical adsorbtion (for a review see [8]).

The derived gap anisotropy function (4.9) and its interpolation (4.11) compared to the ARPES experiment showed that the "standard" four-band model spanned on the $\mathrm{Cu} 3 \mathrm{~d}_{x^{2}-y^{2}}, \mathrm{Cu} 4 \mathrm{~s}, \mathrm{O} 2 \mathrm{p}_{x}$, and O $2 \mathrm{p}_{y}$ orbitals, with an antiferromagnetic s-d pairing interaction, successfully describes the main features of the ARPES data: the roundedsquare-shaped Fermi surface, small energy dispersion along the $(0,0)-(2 \pi, 0)$ line, and the d-type $\left(B_{1 g}\right)$ symmetry of the energy gap $\Delta_{p}$ along the Fermi contour. According to the pairing scenario proposed here, strong electron correlations "drive" the electron exchange amplitudes. These inter- and intra-atomic processes occur on energy scales unusually large for solid state physics. However, the subsequent treatment of the lattice Hamiltonian can be performed completely within the framework of the traditional BCS theory. The criterion for applicability of the BCS scheme is not given by the $J$ vs $t$, but rather by the $T_{\mathrm{c}}$ vs $E_{F}-\epsilon_{\mathrm{b}}$ relation. Taking into account the typical ARPES-derived bandwidths, which are much bigger than $T_{\mathrm{c}}$ we come to the conclusion that the BCS trial wave function [120] is applicable for the description of superconductivity in the layered cuprates with an acceptable accuracy if $T_{\mathrm{c}}$ does not significantly exceed room temperature.

It is worth adding also a few remarks on the normal properties of the layered cuprates. Among all debated issues in the complex physics of the cuprates, the most important one is perhaps that of the normal-phase kinetics. The long-standing problem is whether the paring interaction dominates (or totally determines) the mechanism of Ohmic resistance in the normal phase, as is the case for conventional superconductors. Within the present theory this question can be formulated as follows: does the s-d exchange interaction dominate the scattering of the normal-state charge carriers above $T_{\mathrm{c}}$ ? This is a solvable kinetic problem whose rigorous treatment will be given elsewhere. Here we shall restrain ourselves in providing only a qualitative discussion.

In electron-electron scattering, just like in traffic accidents, the crucial effect comes from the backscattering in "head-on" collisions. For backscattering (i.e., $\vartheta=\pi$ ) in the case of s-d interaction, it turns out that the matrix elements entering the pairing amplitude are also important. It can be easily realized that this amplitude vanishes along the diagonals of the Brillouin zone $(0,0)-(\pi, \pi)$ (the $\Gamma$-M direction). Thereby the cold spots on the Fermi contour correspond to the zeros of $\Delta_{p}$. And vice versa, the hot spots are associated with a maximum gap along $(\pi, \pi)-(0, \pi)$ (the M-X direction). In this sense, cuprates repeat the qualitative feature of the conventional superconductors, with a maximal gap corresponding to maximal scattering on the Fermi surface.

All layered cuprates are strongly anisotropic and two-dimensional models give a reasonable starting point to analyze the related electronic processes. Most importantly, the picture of a layered metal brings in something qualitatively new which does not exist for a bulk metal - the "interstitial" electric field between the layers, like the one in any plane capacitor. The thermodynamic fluctuations of this electric field and related fluctuations of the electric potential and charge density constitute an intensive scattering mechanism analogous to the blue-sky mechanism of light scattering by density fluctuations. It has recently been demonstrated [121] that the experimentally observed linear resistance can be rationalized in terms of the plane capacitor scenario; density fluctuations in the layered conductors are more important than the nature of the interaction. In such a way the linear normal-state resistivity is an intrinsic property [121] of the "layered" electron gas and cannot be used as an argument in favor of non-Fermi-liquid behavior. The resistance of the normal phase 
may not be directly related to the pairing mechanism and these problems can be solved separately. Nevertheless it will be interesting to check whether the anisotropic scattering in cuprates [122-124] can be explained within the framework of the s-d pairing Hamiltonian.

The present theory can also predict a significant isotope effect in the cuprates. Even though the $J_{\text {sd }}$ pairing amplitude does not depend on the atomic mass, the charge carriers reside the ionic $\mathrm{CuO}_{2} 2 \mathrm{D}$ lattice, thereby rendering polaron effects, as in any ionic crystal, possible. For the lighter oxygen isotope the lattice polarization is more pronounced, leading to enhanced effective mass and density of states, and reducing the transfer integrals. Overall, the isotope effect in the $\mathrm{CuO}_{2}$ plane is due to the isotope effect of the density of states. This rationale can be quantitatively substantiated. In our theory, upon isotope substitution at $T=0$, e.g., the change of the penetration depth would be mainly driven by the Cooper pair effective mass that could be determined by means of the Bernoulli effect. At that the superfluid density remains unchanged. The calculation of the isotope effect on $T_{\mathrm{c}}$ requires an evaluation of the polaron effects on the conduction band. Although this is a feasible problem, it is beyond the scope of the present work.

The proposed mechanism for pairing in the $\mathrm{CuO}_{2}$ plane can be handled much like an "Alice-in-Wonderland" toy-model, but we find it fascinating that all ingredients of our theory are achievements of quantum mechanics dating back to the memorable 1920s, presently described in every physical textbook, and constituting the fundamentals of solid state physics $[125,126]$. It would be worthwhile attempting to apply the approach, used in this paper, for modelling triplet and heavy-fermion superconductivity as well.

\subsection{Conclusions: the reason for the success of the $\mathrm{CuO}_{2}$ plane}

We find it very instructive to analyze qualitatively the reasons for the success of the realization of high- $T_{\mathrm{c}}$ superconductivity in the $\mathrm{CuO}_{2}$ plane:

(i) Because of the relatively narrow quasi-two-dimensional conduction d-band, due to p-d hybridization, the density of states is rather high. The wide s-band resulting from s-p hybridization is completely empty, which is somewhat unusual for compounds containing transition ions.

(ii) The pairing s-d exchange process was known since the first years of quantum physics. It is omnipresent in the physics of the transition ions but in order for it to become the pairing mechanism in perovskites it is necessary that the s- and d-levels be close. In other words, a virtual population of the s-level is at least needed in order to make the $J_{\text {sd }}$ amplitude operative. Indeed, the conduction dband is, actually, a result of the s-p-d hybridization in the two-dimensional $\mathrm{CuO}_{2}$ plane.

With the above remarks, one can speculate that among the perovskites the layered ones are more favorable for achieving higher $T_{\mathrm{c}}$ (cf. also the discussion in the Appendix on page 26). The transition ion series ends with $\mathrm{Cu}^{2+}$ and the $\mathrm{Cu} 3 \mathrm{~d}_{x^{2}-y^{2}}$ and 4 s levels are too close. One should keep in mind that the filling of the electron shells finishes with a "robbery" in $\mathrm{Cu}$ [52]: $3 \mathrm{~d}^{10} 4 \mathrm{~s}^{1}$ instead of $3 \mathrm{~d}^{9} 4 \mathrm{~s}^{2}$ as one could expect from the electron configuration of the $\mathrm{Ni}$ atom $\left(3 \mathrm{~d}^{8} 4 \mathrm{~s}^{2}\right)$. However, the energy difference between these two $\mathrm{Cu}$ shell configurations is very small. 
Another favorable factor is the proximity of the $\mathrm{O} 2 \mathrm{p}$ and $\mathrm{Cu} 3 \mathrm{~d}_{x^{2}-y^{2}}$ levels. Thus, post factum the success of $\mathrm{Cu}$ and $\mathrm{O}$ looks quite deterministic: the $\mathrm{CuO}_{2}$ plane is a tool to realize a narrow d-band with a strong s-p-d hybridization. It was mentioned earlier that $J_{\mathrm{sd}}$ is one of the largest exchange amplitudes, but the $4 \mathrm{~s}$ and $3 \mathrm{~d}$ orbitals are orthogonal and necessarily require an intermediary whose role is played by the $\mathrm{O} 2 \mathrm{p}$ orbital. Hence this theory can be nicknamed "the 3d-to-4s-by-2p highway to superconductivity" [19]. The $J_{\mathrm{sd}}$ amplitude is omnipresent for all transition ion compounds, the hybridization of $3 \mathrm{~d}, 4 \mathrm{~s}$ and $2 \mathrm{p}$ is however specific only for the $\mathrm{CuO}_{2}$ plane.

How this qualitative picture can be employed to predict new superconducting compounds is difficult to assess immediately. We believe, however, that this picture, working well for the overdoped regime, is robust enough against the inclusion of all the accessories inherent to the physics of optimally doped and underdoped cuprates: cohabitation of superconductivity and magnetism [95], stripes [127], pseudo-gap [128], interplay of magnetism and superconductivity at individual impurity atoms [129], apex oxygen, $\mathrm{CuO}_{2}$ plane dimpling, doping in chains [130], the $41 \mathrm{meV}$ resonance [131], etc. Perhaps some of these ingredients can be used in the analysis of triplet superconductivity in the copper-free layered perovskite $\mathrm{Sr}_{2} \mathrm{RuO}_{4}$ [132]. It is also likely that the superconductivity of the $\mathrm{RuO}_{2}$ plane is a manifestation of a ferromagnetic exchange integral $J$. The two-electron exchange mediates superconductivity and magnetism in heavy Fermion compounds [133] as well. We suppose that lattice models similar to the approach here will be of use in revealing the electronic processes in these interesting materials. Two-electron exchange may even contribute to the $30 \mathrm{~K} T_{\mathrm{c}}$ of the cubic perovskite $\mathrm{Ba}_{0.6} \mathrm{~K}_{0.4} \mathrm{BiO}_{3}$ but so far it is difficult to separate the exchange contribution from the phonon part of the pairing interaction. However, the strange doping behaviour of $\mathrm{Tl}_{2} \mathrm{Ba}_{2} \mathrm{CuO}_{6 \pm \delta}$ in comparison with $\mathrm{YBCO}$ requires more detailed investigation [11].

\subsection{Perspectives: if "Tomorrow" comes...}

The technological success in preparing the second generation of high- $T_{\mathrm{c}}$ superconducting cables by depositing thin-layer superconducting ceramics on a flexible low-cost metallic substrate is crucial for the future energy applications. The USA Department of Energy suggests global superconducting energy products would command an annual market of $30 \mathrm{G} \$$ by about 2020 . High- $T_{\mathrm{c}}$ superconductor power cables, transformers, motors and generators could grab a 50\% market share by 2013, 2015, 2016 and 2021, respectively [134]. On the other hand atomic-layer engineering of superconducting oxides will trigger progress in materials science and electronics. One can envision multi-functional all-oxide electronics, e.g., sensors, processing and memory devices, all monolithically integrated within a single chip [135]. In spite of the technological progress and tens of thousands of publications the theoretical "picture in early 2000 remains fairly cloudy on the whole" [2]. The landmark of "Today" must be some event. "What event will it be? It is desirable that this landmark be the insight into the mechanism of superconductivity in high- $T_{\mathrm{c}}$ cuprates" [2].

In this paper we presented a traditional theory for superconductivity in overdoped, and possibly also optimally doped cuprates. All of its ingredients can be found in the textbooks and there is a considerable chance that we witness the victory of traditionalism, as it was in the history of quantum electrodynamics (QED) half a century ago, but it may well be just a personal viewpoint "brainwashed by 
Feynman" [136]. Nonetheless let us use the example of QED to illustrate the essence of our contribution. QED appeared as a synthesis between perturbation theory and relativity. Both components had been known well before the QED conception. Similarly, both the BCS theory and the exchange interaction have been known for ages, so the point in the agenda was how to conceive out of them the theory of high- $T_{\mathrm{c}}$ cuprates. Such a theory contains necessarily a big number of energy parameters $\left(E_{\mathrm{F}}, \epsilon_{\mathrm{s}}, \epsilon_{\mathrm{p}}, \epsilon_{\mathrm{d}}, t_{\mathrm{sp}}, t_{\mathrm{pp}}, t_{\mathrm{pd}}, J_{\mathrm{sd}}, J_{\mathrm{pd}}, J_{\mathrm{sp}}, J_{\mathrm{pp}}\right)$ which are difficult to determine simultaneously (for the current status of the problem see for example reference $[137,138])$. The first step will definitely be to use ARPES data in which the spectrum is clearly seen and to neglect in a first approximation the "irrelevant" inter-atomic exchange integrals $J_{\mathrm{pd}}, J_{\mathrm{sp}}$ and $J_{\mathrm{pp}}$. In this case, for a known normal spectrum one can determine $J_{\mathrm{sd}}$ from $T_{\mathrm{c}}$ or from the maximum gap at $T=0$.

A crucial "meeting point" between theory and experiment is the GinzburgLandau (GL) theory. The general form of the GL coefficients for anisotropic-gap superconductors, including the effect of disorder, is given in reference [139] and is directly applicable to the present model. For the s-d separable kernel (4.4) the specific heat $C(T)$ in the clean limit can also be explicitly derived [140] and has the GL form $C(T)=C_{\mathrm{N}}+C_{\Delta}$, with

$$
\begin{aligned}
& C_{\mathrm{N}}(T)=\frac{\pi^{2}}{3}\left\langle q_{c}\left(\nu_{\boldsymbol{p}}\right)\right\rangle, \\
& C_{\Delta}(T)=k_{\mathrm{B}} T \frac{\alpha^{2}}{b}=\frac{4 \pi^{2}}{7 \zeta(3)} \frac{\left\langle\chi_{\boldsymbol{p}}^{2} q_{a}\left(\nu_{\boldsymbol{p}}\right)\right\rangle^{2}}{\left\langle\chi_{\boldsymbol{p}}^{4} q_{b}\left(\nu_{\boldsymbol{p}}\right)\right\rangle} \theta\left(T_{\mathrm{c}}-T\right),
\end{aligned}
$$

where $\nu_{p}=\frac{E_{p}}{2 k_{\mathrm{B}} T}$, and

$$
\begin{aligned}
& \alpha(T)=\frac{1}{2\left(k_{\mathrm{B}} T\right)^{2}}\left\langle\chi_{\boldsymbol{p}}^{2} q_{a}\left(\nu_{\boldsymbol{p}}\right)\right\rangle, \quad b(T)=\frac{7 \zeta(3)}{16 \pi^{2}\left(k_{\mathrm{B}} T\right)^{3}}\left\langle\chi_{\boldsymbol{p}}^{4} q_{b}\left(\nu_{\boldsymbol{p}}\right)\right\rangle, \\
& q_{a}(\nu)=\frac{1}{2 \cosh ^{2} \nu}, \quad q_{b}(\nu)=\frac{\pi^{2}}{14 \zeta(3)} \frac{1}{\nu^{2}}\left(\frac{\tanh \nu}{\nu}-\frac{1}{\cosh ^{2} \nu}\right), \quad q_{c}(\nu)=\frac{6}{\pi^{2}} \frac{\nu^{2}}{\cosh ^{2} \nu} .
\end{aligned}
$$

Accordingly, the jump of the specific heat at $T_{\mathrm{c}}$ is expressed by the GL coefficients $\alpha$ and $b, \Delta C=k_{\mathrm{B}} T_{\mathrm{c}} \alpha^{2}\left(T_{\mathrm{c}}\right) / b\left(T_{\mathrm{c}}\right)$. With the help of the general equations (6.6) one can further determine the influence of the Van Hove singularity on the thermodynamic and electrodynamic behaviour. For $\Delta C$ the effect of the Van Hove singularity is reported in reference [141], and for a general review on the Van Hove scenario of high$T_{\mathrm{c}}$ superconductivity we refer the reader to reference [142]. When the Fermi level is not close to the Van Hove singularity the GL coefficients can be worked out as integrals over the Fermi surface; methodological details are given in reference [143]. Knowledge of the GL coefficients is also fundamental for the physics of fluctuation phenomena in superconductors [144].

Furthermore, a microscopic consideration of the London penetration depth $\lambda$ for screening currents in the $\mathrm{CuO}_{2}$ plane gives [145]

$$
\frac{1}{\lambda^{2}(T)}=\frac{e^{2}}{\varepsilon_{0} c^{2} \hbar^{2} d_{\mathrm{eff}}} \oint v_{\boldsymbol{p}} r_{d}\left(\nu_{\boldsymbol{p}}\right) \frac{\mathrm{d} p_{l}}{(2 \pi)^{2}},
$$

$\ddagger$ The gap-anisotropy fit in figure $3(\mathrm{~d})$ is quite robust against the choice of the parameters. To illustrate and emphasize the capability of the model we have used, for example, unrealistically big values of the hopping integrals: $\varepsilon_{\mathrm{d}}=0, \varepsilon_{\mathrm{s}}=5, \varepsilon_{\mathrm{p}}=-0.9, t_{\mathrm{pd}}=1.13, t_{\mathrm{sp}}=1.63, t_{\mathrm{pp}}=0.2 \mathrm{eV}$. This set of parameters corresponds to band calculations but gives a factor $2-3$ wider conduction band. If the band is fitted to the ARPES data $J_{\text {sd }}$ can be less than $1 \mathrm{eV}$. A realistic fit is deemed to be a subject of a collaboration with experimentalists. 


$$
r_{d}(\nu)=\nu^{2} \sum_{n=0}^{\infty}\left[\nu^{2}+\pi^{2}(n+1 / 2)^{2}\right]^{-3 / 2},
$$

where the integration is performed along the Fermi contour. The penetration depth $\lambda(T)$ is involved in the Bernoulli effect in superconductors [146]:

$$
\frac{\Delta \varphi}{\mathcal{R}_{\mathrm{H}}}=-\frac{e^{2}}{2 \varepsilon_{0} c^{2}} \lambda^{2}(T) j^{2}, \quad \frac{1}{\mathcal{R}_{\mathrm{H}}}=\frac{2|e|}{a_{0}^{2} d_{\mathrm{eff}}} \oint p_{y}\left(p_{x}\right) \frac{\mathrm{d} p_{x}}{(2 \pi)^{2}},
$$

where $\Delta \varphi$ is the change of the electric potential induced by a current density $\mathbf{j}$, $1 / \mathcal{R}_{\mathrm{H}}=e n_{\text {tot }}$ is the volume charge density of the charge carriers, with $d_{\text {eff }}$ the effective spacing between the $\mathrm{CuO}_{2}$ planes.

For given penetration depth extrapolated to zero temperature, $\lambda(0)$, and Hall constant of the superconducting phase, one can easily determine the effective mass of the Cooper pairs

$$
m^{*}=\frac{e^{*} \lambda^{2}(0)}{\varepsilon_{0} c^{2} \mathcal{R}_{\mathrm{H}}}, \quad\left|e^{*}\right|=2|e| .
$$

This important material parameter $m^{*}$ is experimentally accessible from the electrostatic modulation of the kinetic inductance of thin superconducting films [147] as well as from the surface Hall effect [148].

Having a big variety of calculated variables the parameters of the theory can be reliably fitted. Another research direction is the first-principles calculation of the transfer amplitudes and two-electron exchange integrals. The level of agreement with the fitted values will be indicative for the completeness of our understanding. In addressing more realistic problems, the properties of a single space-homogeneous $\mathrm{CuO}_{2}$ plane will be a reasonable starting point. Concluding, we believe that there is a true perspective for the theoretical physics of cuprate superconductors to become an important ingredient of their materials science.

Magnetism and superconductivity are among the most important collective phenomena in condensed matter physics. And, remarkably, magnetism of transition metals and high- $T_{\mathrm{c}}$ superconductivity of cuprates seem to be two faces of the same ubiquitous two-electron exchange amplitude.

\section{Acknowledgments}

This work was supported by the Flemish GOA. This work is dedicated in memoriam to our colleague and friend A V Groshev who was an enthusiastic collaborator in the early stages of this years-long endeavour. T M M is much indebted to T Sariisky for the stimulating discussions in the course of the pre-cuprates era seminars on the problem of high- $T_{\mathrm{c}}$ superconductivity held in Sofia in the early 1980s. T M M is thankful to D Damianov for partial financial support. The realization of this work would have been impossible without the cooperation of V Mishonova. We are also indebted to Prof M Mateev for his continuous support during this long research and to Prof B Bioltchev for the support in the final stages of this project. It is a pleasure to acknowledge extensive comments and correspondence by (in chronological order) P B Littlewood, J Zaanen, C Di Castro, N M Plakida, J M J van Leeuwen, J de Jongh, P-G de Gennes, S Sachdev, Ph Nozières, C M Varma, V L Pokrovsky, P Wiegmann, B L Altschuler, A Varlamov, A Rigamonti, F Borsa, F H Read, M Mateev, P Brovetto, M Sigrist, J Bouvier, D Damianov, L P Pitaevskii, J Friedel, J Bok, M Mishonov, D Markowitz and L P Kadanoff. 


\section{Appendix. $T_{\mathrm{c}}-\epsilon_{\mathrm{S}}$ correlations: a hint toward the mechanism of superconductivity in cuprates}

In the letter by Pavarini et al, reference [149], a strong correlation is observed between $T_{c \max }$ and a single parameter

$$
s(\epsilon)=\left(\epsilon_{\mathrm{s}}-\epsilon\right)\left(\epsilon-\epsilon_{\mathrm{p}}\right) /\left(2 t_{\mathrm{sp}}\right)^{2},
$$

which is controlled by the energy of the $\mathrm{Cu} 4 \mathrm{~s}$ orbital $\epsilon_{\mathrm{s}}, \epsilon_{\mathrm{p}}$ is the $\mathrm{O} 2 \mathrm{p}$ single cite energy, $t_{\mathrm{sp}}$ is the transfer integral between neighbor $\mathrm{Cu} 4 s$ and $\mathrm{O} 2 p$ orbitals, and $\epsilon_{\boldsymbol{p}}$ is the conduction band energy whose bottom at the $\Gamma$ point coincides with the $\mathrm{Cu}$ $3 \mathrm{~d}_{x^{2}-y^{2}}$ energy $\epsilon_{\mathrm{d}}$. It is unfortunate that theorists have not so far paid any attention to this observation because it is an important correlation between the ab initio calculated parameter $r=(1+s) / 2$ and the experimentally measured $T_{\mathrm{c} \text { max }}$ which can reveal the subtle link between the experiment and the theory and finally solve the long-standing puzzle of the mechanism of high- $T_{\mathrm{c}}$ superconductivity (HTSC).

The purpose of the present Comment [150] is to emphasize that the missing link has already been found, and the work by Pavarini et al can be used as a crucial test for theoretical models of HTSC. Perhaps the simplest possible interpretation, though one could search for alternatives, is given within the framework of the present theory. In order for the Schubin-Zener-Kondo exchange amplitude $J_{\text {sd }}$ to operate as a pairing interaction of the charge carriers, it is necessary the $\mathrm{Cu} 4 \mathrm{~s}$ orbital to be significantly hybridized with the conduction band. The degree of this hybridization depends strongly on the proximity of the $\mathrm{Cu} 4 \mathrm{~s}$ level to the Fermi level $\epsilon_{\mathrm{F}}$. Thus, it is not surprising that $\epsilon_{\mathrm{S}}$ controls the maximal critical temperature $T_{\mathrm{c} \text { max }}$, being the only parameter of the $\mathrm{CuO}_{2}$ plane which is essentially changed for different cuprate superconductors.

$\mathrm{Cu} 3 \mathrm{~d}$ and $\mathrm{Cu} 4 \mathrm{~s}$ are orthogonal orbitals and their hybridization is indirect. First, the $\mathrm{Cu} 3 \mathrm{~d}_{x^{2}-y^{2}}$ orbital hybridizes with the $\mathrm{O} 2 \mathrm{p}_{x}$ and $\mathrm{O} 2 \mathrm{p}_{y}$ orbitals, then the $\mathrm{O} 2 \mathrm{p}$ orbitals hybridize with $\mathrm{Cu} 4 \mathrm{~s}$. $\mathrm{As}$ a result we have a "3d-to-4s-by-2p" hybridization of the conduction band of HTSC cuprates which makes it possible the strong antiferromagnetic amplitude $J_{\text {sd }}$ to create pairing in a relatively narrow $\mathrm{Cu} 3 \mathrm{~d}_{x^{2}-y^{2}}$ conduction band. The hybridization "filling" of the $\mathrm{Cu} 4 \mathrm{~s}$ orbitals can be seen in cluster calculations as well [151]. The $s(\epsilon)$ parameter introduced in reference [149] reflects the proximity of all 3 levels in the generic 4-band Hamiltonian of the $\mathrm{CuO}_{2}$ plane - their "random coincidence" for the $\mathrm{Cu}-\mathrm{O}$ combination. Suppose that those levels are not so close to each other. In this case the slightly modified parameter

$$
s^{\prime}(\epsilon)=\left(\epsilon_{\mathrm{s}}-\epsilon\right)\left(\epsilon-\epsilon_{\mathrm{p}}\right) /\left(4 t_{\mathrm{sp}} t_{\mathrm{pd}}\right)
$$

is simply the energy denominator of the perturbation theory which describes the hybridization filling of the axial orbital, see (4.10). Whence $\left[s^{\prime}(\epsilon)\right]^{2}$ is a denominator of the pairing amplitude in the BCS equation (6.4). Hence, we conclude that the correlations reported in reference [149] are simply the correlations between the critical temperature $T_{\mathrm{c}}$ and the dimensionless BCS coupling constant $\rho\left(\epsilon_{\mathrm{F}}\right) J_{\mathrm{sd}} /\left[s^{\prime}\left(\epsilon_{\mathrm{F}}\right)\right]^{2}$. Of course, for coupling constants $\sim 1$ the BCS trial wavefunction can be used only for qualitative estimates, but knowing the Hamiltonian the mathematical problem may somehow be solved. In any case, even qualitatively, we are sure that the stronger pairing amplitude $J_{\mathrm{sd}}$ and hybridization $1 / s^{\prime}\left(\epsilon_{\mathrm{F}}\right)$ enhance $T_{\mathrm{c}}$.

Having LDA calculations for the band structures of many cuprates it is worthwhile performing a LCAO fit to them [64] and using experimental values of $T_{\mathrm{c}}$ to extract 
the pairing amplitude $J_{\mathrm{sd}}$ for all those compounds. The ab initio calculation of the Kondo scattering amplitude parameterized by $J_{\text {sd }}$ is an important problem which has to be set in the agenda of computational solid state physics. We expect that it will be a weakly material dependent parameter of the order of the s-d exchange amplitude in Kondo alloys, but perhaps slightly bigger as for the $\mathrm{Cu}$ ion the $3 \mathrm{~d}$ and $4 \mathrm{~s}$ levels are closer compared to many other ions. Closer energy levels, from classical point of view, imply closer classical periods of orbital motion which leads to some "resonance" enhancement of the exchange amplitude due to intra-atomic two-electron correlations. The final qualitative conclusion that can be extracted from the correlations reported by Pavarini et al is the explanation why only the $\mathrm{CuO}_{2}$ plane renders HTSC possible, whereas hundreds other similar compounds are not even superconducting, or have only a "conventional" value of $T_{\mathrm{c}}$. The natural explanation is: because its $s$-parameter is not small enough below its critical value. Even among the cuprates one can find compounds with "conventional" values of $T_{\mathrm{c}}$ having relatively large value of the $s$ parameter. For other transition metal compounds the parameter

$$
s^{\prime}\left(\epsilon_{\mathrm{d}}\right)=\left(\epsilon_{\mathrm{s}}-\epsilon_{\mathrm{d}}\right)\left(\epsilon_{\mathrm{d}}-\epsilon_{\mathrm{p}}\right) /\left(4 t_{\mathrm{sp}} t_{\mathrm{pd}}\right)
$$

is much bigger than its critical value $s_{c}$ which can be reached probably only for the $\mathrm{Cu}-\mathrm{O}$ combination. Thereby, the correlation reported by Pavarini et al is a crucial hint which of the models for HTSC is still on the arena. Concluding, we also note that the $4 \mathrm{~s}$ hybridization is responsible for the three-dimensional coherent Fermi surface of $\mathrm{Tl}_{2} \mathrm{Ba}_{2} \mathrm{CuO}_{6+\delta}[152]$

\section{References}

[1] Bednorz J G and Müller K A 1986 Z. Phys. B 64 189-193

Bednorz J G and Müller K A 1988 Rev. Mod. Phys. 60 585-600

[2] Ginzburg V L 2000 J. Supercond.: Incorp. Novel Magn. 13 665-677

[3] Cooper L N 1956 Phys. Rev. 104 1189-1190

Bardeen J, Cooper L N and Schrieffer J 1957 Phys. Rev. 106 162-4

Bardeen J, Cooper L N and Schrieffer J 1957 Phys. Rev. 108 1175-1204

[4] Aebi P 2001 Fermi surface mapping by Angle-Scanned Photoemission, in High-Tc Superconductors and Related Materials: Materials Science, Fundamental Properties, and Some Future Electronic Applications ed S-L Drechsler and T M Mishonov (Dordrecht: Kluwer) pp 39-50

[5] Campuzano J C, Norman M R and Randeria M 2002 Photoemission in the high- $T_{\mathrm{C}}$ superconductors, to appear in The Physics of Superconductors ed K-H Bennemann and J P Ketterson (Heidelberg: Springer) vol 2

Campuzano J C, Norman M R and Randeria M 2002 Preprint cond-mat/0209476

[6] Scalapino D J 1995 Phys. Rep. 250 329-367

Poilblanc D and Scalapino D 2002 Calculation of $\Delta(k, \omega)$ for a $2 \mathrm{D} t$-J cluster Preprint cond-mat/0202180

[7] Schrieffer J R 1995 J. Low Temp. Phys. 9997

[8] Plakida N M 1995 High-Temperature Superconductivity (Berlin: Springer-Verlag)

[9] Annett J F, Goldenfeld N and Leggett A J 1996 J. Low Temp. Phys. 105473 Lee P A 1996 J. Low Temp. Phys. 105581

[10] Koltenbah B E C and Joynt R 1996 Rep. Prog. Phys. 60 23-56 Li Q P, Koltenbah B E C and Joynt R 1993 Phys. Rev. B 48437

[11] Ruvalds J 1996 Supercond. Sci. Technol. 9 905-926

[12] Markiewicz R S 1997 J. Phys. Chem. Solids 58 1179-1310

[13] Brusov P 1999 Mechanisms of High Temperature Superconductivity, in 2 volumes, (Rostov: Rostov State University Publishing) 2815 references, 701 pages.

[14] Wilson J A 2000 J. Phys.: Condens. Matter 12 R517-R547

Wilson J A 2001 J. Phys.: Condens. Matter 13 R945-R977

Wilson J A 2003 Preprint cond-mat/0304661 
[15] Szotek Z, Györffy B L, Temmerman W M, Andersen O K and Jepsen O 2001 J. Phys.: Condens. Matter 13 8625-8652

[16] Chubukov A V, Pines D and Schmalian J 2003 A spin fluctuation model for d-wave superconductivity, in The Physics of Superconductors ed K-H Bennemann and J B Ketterson (Heidelberg: Springer) vol 1

Chubukov A V, Pines D and Schmalian J 2002 Preprint cond-mat/0201140

Plakida N M, Anton L, Adam S and Adam Gh 2002 Exchange and spin-fluctuation pairing in cuprates Preprint cond-mat/0104234

Plakida N M, Hayn R and Richard J-L 1995 Phys. Rev. B 5216599

Friedel J and Kohmoto M 2002 Eur. Phys. J. B $30427-435$

[17] Carlson E W, Emery V J, Kivelson S A and Orgad D 2002 Concepts in high-temperature superconductivity, in The Physics of Superconductors ed K-H Bennemann and J B Ketterson (Heidelberg: Springer) vol 1

Carlson E W, Emery V J, Kivelson S A and Orgad D 2002 Preprint cond-mat/0206217

[18] Rigamonti A, Borsa F and Carretta P 1998 Rep. Prog. Phys. 61 1367-1439

[19] Mishonov T M, Indekeu J O and Penev E S 2002 The 3d-to-4s-by-2p highway to superconductivity in cuprates Int. J. Mod. Phys. B 16 4577-4585

(Preprint cond-mat/0206350)

[20] Schubin S and Wonsowsky S 1934 Proc. R. Soc. London Ser. A 145 159-180

Vonsovskii S V 1946 Zh. Eksper. Teor. Fiz. 16980

Zener C 1951 Phys. Rev. 81 440-444

Zener C 1951 Phys. Rev. 82 403-405

Zener C 1951 Phys. Rev. 83 299-301

Vonsovskii S V 1974 Magnetism (New York: John Wiley \& Sons)

Vonsovskii S V, Izyumov Yu A and Kurmaev E Z 1982 Superconductivity of Transition Metals, Their Alloys and Compounds (Berlin: Springer)

Craik D 1995 Magnetism: Principles and Applications (Chichester: Wiley)

Yosida K 1996 Theory of Magnetism (Berlin: Springer) p 181, p 319

Yosida K 1966 On s-d and s-f Interactions, in Magnetism ed G T Rado and H Suhl (San Diego: Academic Press) vol IIB, pp 215-291

Schubin S P 1991 Selected Papers on Theoretical Physics ed S V Vonsovskii and M I Katsnelson (Ekaterinburg: Ural branch RAS) p 375 (in Russian); Schubin died in a lager in 1938, cf. Vizgin V P 1999 The nuclear shield in the 'thirty-year war' of physicists against ignorant criticism of modern physical theories Phys. Usp. 42 1259-1283

Andreev A F et al 1999 In memory of Sergei Vasil'evich Vonsovskii Sov. Phys. Usp. 42 101-102 Izumov Yu A 2002 Basic Models in Quantum Theory of Magnetism (Ekaterinburg: Ural branch RAS)

See also Bogoliubov N N 1967 Selected works. Vol 1. Lectures on quantum statistics (New York: Gordon and Breach Science Publishers); (Kiev: Naukova Dumka, 1970)(in Russsian); (Kiev: Ryadanskaya Shkola, 1948)(in Russian); It is interesting to note that in the 1948 lectures Bogoliubov has implicitly introduced what is now known as the Hubbard model, as well as referred to Vonsovskii's works.

[21] Heitler W and London F 1927 Z. Phys. 44445

[22] Mishonov T M, Donkov A A, Koleva R K and Penev E S 1997 Superconducting gap anisotropy within the framework of a simple exchange model for layered cuprates. The theory of HTSC Bulgarian J. Phys. 24 114-125

(Preprint cond-mat/0001033)

[23] Mishonov T M, Groshev A V and Donkov A A 1998 Bulgarian J. Phys. 2562

[24] Mishonov T M, Wallington J P, Penev E S and Indekeu J O 2002 Mod. Phys. Lett. B 16693

[25] Bloch F 1928 Z. Phys. 72555

[26] Hückel E 1927 Z. Phys. 70204

Hückel E 1932 Z. Phys. 76628

[27] Slater J C 1963 Quantum Theory of Molecules and Solids (New York: McGraw-Hill) vol 1, section 2.3, chap 3, chap 9 and vol 2

[28] Labbé J and Bok J 1987 Europhys. Lett. 3 1225-1230

[29] Mishonov T and Penev E 2000 Tight-binding modelling of the electronic band structure of layered superconducting perovskites J. Phys.: Condens. Matter 12 143-159

(Preprint cond-mat/0001049)

[30] Heisenberg W 1926 Z. Phys. 38411

Heisenberg W 1928 Z. Phys. 49619

[31] Vonsovskii S V and Svirskii M S 1960 Zh. Eksper. Teor. Fiz. 39384 [1961 Sov. Phys.-JETP 
12 272-277]

Morkovskii Ya 1962 Fiz. Met. Metalloved. 13940

Sarma G J 1963 J. Phys. Chem. Solids 241029

Vonsovskii S V and Svirsky M S 1965 phys. stat. sol. 9 267-280

Dunin S Z 1972 Sov. Phys. Solid State 14 559-560

Izyumov Yu A and Skryabin Yu N 1974 phys. stat. sol. (b) 61 9-64

Bennemann K-H and Garland J W 1979 Int. J. Magnetism 197

Lee T-K, Izyumov Yu A and Birman J L 1979 Phys. Rev. B 204494

Tonchev N S and Brankov I G 1980 phys. stat. sol. (b) 102179

Izyumov Yu A 1991 Sov. Phys. Usp. 34 935-957

[32] Nielsen J R (ed) 1976 Niels Bohr, Collected Works (New York: North-Holland) in 3 volumes

Bohr N 1913 Philos. Mag. 26 1-25

Bohr N 1913 Philos. Mag. 26 476-502

Born M 1925 Vorlesungen über Atommechanik (Berlin: Springer) p 331, figure 40

[33] Langmuir I 1921 Phys. Rev. 17339

[34] van Vleck J H 1922 Philos. Mag. 44842

[35] Read F H 1982 Aust. J. Phys. 35 475-499

Read F H 1977 J. Phys. B: Atom. Molec. Phys. 10 449-458

Brunt N H, Read F H, King G C 1977 J. Phys. E: Scientific Instruments 10 134-139

Buchman S J, Hammond P, Read F H and King G C 1983 J. Phys. B: Atom. Molec. Phys. 16 4039-4047

Read F H 1985 Threshold behaviour impact ionization, in Electron Impact Ionization ed T D Märk and G H Dunn (New York: Springer) pp 42-88

Read F H 1990 J. Phys. B: At. Mol. Opt. Phys. 23 951-958

[36] Thomson J J 1914 Philos. Mag. 27 757-789

[37] Blackemore J 1985 Solid State Physics 2nd edition (Cambridge: Cambridge University Press) section 3.2

[38] Ogg R A 1946 Phys. Rev. 69243

[39] Schafroth M R 1955 Phys. Rev. 100 463-475

[40] Lewis G N 1916 J. Am. Chem. Soc. 38 762-786

[41] Langmuir I 1919 J. Am. Chem. Soc. 41868

Langmuir I $1919 \mathrm{~J}$. Am. Chem. Soc. 411543

[42] Parson A L, A magneton theory of the structure of the atom Smithsonian Miscellaneous Collection vol 65, publication No 2371, pp 1-80

[43] Lewis G N 1926 Nature 118 874-875

[44] Wilson S 1984 Electron Correlations in Molecules (Oxford: Clarendon Press) section 7.3

[45] Herring C and Flicker 1964 Phys. Rev. 134 A362-A366

Herring C 1962 Critique of the Heitler-London method of calculating spin couplings at larger distances Rev. Mod. Phys. 34 631-645

Herring C 1966 Direct exchange between well-separated atoms, in Magnetism ed G T Rado and H Suhl (San Diego: Academic Press) vol IIB, p 76

[46] Herring C 1966 Exchange interactions among itinerant electrons, in Magnetism ed G T Rado and H Suhl (San Diego: Academic Press) vol IV

[47] Patil S H and Tang K T 2000 Asymptotic Methods in Quantum Mechanics. Application to Atoms, Molecules and Nuclei (Springer: Berlin)

[48] Dreizler R M and Gross E K U 1990 Density Functional Theory (Springer, Berlin)

Oliveira L N, Gross E K U and Kohn W 1988 Phys. Rev. Lett. 60 2430-2433

Kurth S, Marques M, Lüders M and Gross E K U 1999 Phys. Rev. Lett. 83 2628-2631

[49] Gor'kov L P and Krotkov P L 2002 Preprint cond-mat/0209141

[50] Broveto P, Maxia V and Salis M 2000 Eur. Phys. J. B 17 85-94

[51] Dirac P A M 1929 Proc. R. Soc. London Ser. A 123714

Dalitz R H (ed) 1995 The Collected Works of P. A. M. Dirac (Cambridge: Cambridge University Press) p 389

Dirac P A M 1930 The Principles of Quantum Mechanics (Oxford: Clarendon Press) chap IX: Systems Containing Several Similar Particles

[52] Feynman R P, Leighton R B and Sands M 1966 The Feynman Lectures on Physics (Reading: Addison-Wesley) vol III, equation (12.16), figure 4-15

[53] Schwinger J 2001 Quantum Mechanics: Symbolism of Atomic Measurements ed B G Englert (San Diego: Academic Press) equation (3.5.14), p 160

[54] Harris A B and Lange R V Phys. Rev. 157 295-314

[55] Fulde P 1995 Electron Correlations in Molecules and Solids, 3rd enlarged ed. (Berlin: Springer- 
Verlag)

[56] Spalek J and Honig J M 1991 Metal-insulator transitions, exchange interactions and real space pairing. An introduction to the theory of strongly correlated electron systems, in Studies of High-Temperature Superconductors. Advances in Research and Applications ed A Narlikar (New York: Nova Science) pp 1-67

[57] Ketterson J B and Song S N 1999 Superconductivity (Cambridge: Cambridge University Press) section 26, p 203 and section 33, p 243

[58] Mishonov T and Groshev A 1994 Two-electron exchange between adjacent oxygen atoms as a possible origin of the pairing in layered cuprates Physica B 194-196 1427-1428

[59] Tsuei C C and Kirtley J R 2000 Rev. Mod. Phys. 72 969-1016, figure 4

[60] Markowitz D and Kadanoff L P 1965 Phys. Rev. 131563

[61] Anderson P W 2002 Phys. Scr. T102 10-12

(Preprint cond-mat/0201429)

Here the author writes: ". . . the interlayer tunneling theory which I advocated for five years, and wrote a book about, turns out to be one of those which must be consigned to the dustbin". This voluntary concession marks the end of an epoch of unconventional theories of high- $T_{\mathrm{C}}$ superconductivity. Further, in the same paper, the assumption "without any justification" of the BCS gap equation for "one phonon which can be expected to couple rather strongly..." is a premonition of the return to conventional BCS interpretations of the high- $T_{\mathrm{c}}$ problem.

[62] Pokrovskii V L 1961 Zh. Eksper. Teor. Fiz. 40641 [1961 Sov. Phys.-JETP 13 447]

Pokrovskii V L and Ryvkin M S 1962 Zh. Eksper. Teor. Fiz. 4392 [1963 Sov. Phys.-JETP 16 $67]$

[63] Schwaller P, Aebi P, Berger H, Beeli C, Osterwalder J and Schlapbach L 1995 J. Electron Spectr. Related Phenomena $\mathbf{7 6}$ 127-132

[64] Andersen O K, Lichtenstein A I, Jepsen O and Paulsen F 1995 J. Phys. Chem. Solids 56 $1573-1591$

[65] Mesot J, Norman M R, Ding H, Randeria M, Campuzano J C, Paramekanti A, Kaminski A, Takeuchi T, Yokoya T, Sato T, Takahashi T, Mochiku T and Kadowaki K 1999 Phys. Rev. Lett. 83 840-843, figure 2

[66] Ding H, Norman M R, Campuzano J C, Randeria M, Bellman A F, Yokoya T, Takahashi T, Mochiku T and Kadowaki K 1996 Phys. Rev. B 54 R9678, figure 10

Ding H, Norman M R, Kadowaki K and Giapinzakis J 1996 Nature 382, 51

Randeria M and Campuzano J C 1997 High- $T_{\mathrm{c}}$ superconductors: New insights from AngleResolved Photoemission Preprint cond-mat/9709107, figure 10

Ding H, Campuzano J C, Norman M R, Randeria M, Yokoya T, Takahashi T, Takeuchi T, Mochiku T, Kadowaki K, Guptasarma P and Hinks D G 1998 J. Phys. Chem. Solids 59 1888-1891, figure 1

[67] Abrikosov A A and Falkovsky L A 1990 Physica C 168556

[68] Zeiger H J and Pratt G W 1973 Magnetic Interactions in Solids (Oxford: Clarendon Press) p 53, p 324

[69] Kondo J 1964 Resistance minimum in dilute magnetic alloys Prog. Theor. Phys. 32 37-49 Kondo J 1966 Anomalous scattering due to s-d interaction J. Appl. Phys. 371177

[70] Ruderman M A and Kittel C 1956 Phys. Rev. 9699

Kasuya T 1956 Prog. Theor. Phys. 1645

Yosida K 1957 Phys. Rev. 106893

de Gennes P G 1958 C. R. Acad. Sci. Paris 2471836

de Gennes P G 1962 J. Phys. Radium 23630

Elliott R J 1965, in Magnetism ed G T Rado and H Suhl (San Diego: Academic Press) vol IIA Abrikosov A and Gor'kov L P 1962 Zh. Eksper. Teor. Fiz. 432230

[71] Tsvelick A M and Wiegmann P B 1983 Adv. Phys. 32 453-713

[72] Kramers H A 1934 Physica 1191

Anderson P W 1963 Exchange in insulators: superexchange, direct exchange and double exchange, in Magnetism ed G T Rado and H Suhl (San Diego: Academic Press) vol I

[73] Kondo J $1962 \mathrm{~g}$-shift and anomalous Hall effect in gadolinium metals Prog. Theor. Phys. 28 $846-856$

[74] Anderson P W 1961 Localized magnetic states in metals Phys. Rev. 124 41-53

[75] Friedel J 1956 Can. J. Phys. 34 1190-1211

de Faget de Casteljau P and Friedel J 1956 J. Phys. Radium 17 27-32

Friedel J 1958 J. Phys. Radium 19 573-581

[76] Muto T and Kobayasi S 1964 On indirect Knight shift and NMR in ferromagnetic metals. Part I. General formulation and quantitative discussion J. Phys. Soc. Japan 19 1837-1849 
Muto T, Kobayasi S and Hayakawa H 1965 On indirect Knight shift and NMR in ferromagnetic metals. Part II. Numerical calculation of NMR frequency and hyperfine field in Fe J. Phys. Soc. Japan 20 389-395

Muto T, Kobayasi S and Hayakawa H 1965 On indirect Knight shift and NMR in ferromagnetic metals. Part III. Numerical calculation of NMR frequency and hyperfine field in $\mathrm{Ni}, \mathrm{Co}$ and Fe J. Phys. Soc. Japan 20 1167-1173

[77] Freeman A J and Watson R E 1966 Hyperfine interactions in magnetic materials, in Magnetism ed G T Rado and H Suhl (San Diego: Academic Press) vol IIA, pp 167-305

[78] Ganguly B N 1973 Phys. Rev. B 8 1055-1060

[79] Anderson P W 2002 Preprint cond-mat/0201431; The author concludes that "The simplicity of BCS comes from the simplicity of the Fermi liquid state from which it arises".

[80] Vollhardt D, Blümer N, Held K and Kollar M 2001 Metallic ferromagnetism - an electronic correlation phenomenon, in Band Ferromagnetism: Ground-State and Finite-Temperature Phenomena ed K Baberschke, M Donath and W Nolting, Lecture Notes in Physics (Heidelberg: Springer) vol 580, pp 191-207

Vollhardt D, Blümer N, Held K and Kollar M 2000 Preprint cond-mat/0012203

[81] Sekitani T, Naito M, Sato H and Miura N 2001 Interplay of Cooper and Kondo singlet formations in high- $T_{\mathrm{c}}$ cuprates Preprint cond-mat/0111422 (submitted to Physica C)

[82] Park K 2003 Kondo effect of non-magnetic impurities and the co-existing charge order in the cuprate superconductors Phys. Rev. B 67094513

Park K 2002 Preprint cond-mat/0203142

[83] Kadowaki K and Woods S B 1986 Universal relationship of the resistivity and specific heat in heavy-Fermion compounds Solid State Commun. 58 507-509

[84] Nakamae S, Behnia K, Mangkorntong N, Nohara M, Takagi H, Yates S J C and Hussey N E 2003 Electronic ground state of heavily overdoped nonsuperconducting $\mathrm{La}_{2-x} \mathrm{Sr}_{x} \mathrm{CuO}_{4}$ Phys. Rev. B 68100502

[85] de Nobel J 1996 Phys. Today 49 40-42

[86] Ginzburg V L 1968 The problem of high-temperature superconductivity Contemp. Phys. 9 355-374

Ginzburg V L 1970 The problem of high-temperature superconductivity. II Usp. Fiz. Nauk 101 185-215 [1970 Sov. Phys. Usp. 13 335-351]

Ginzburg V L 1970 High-temperature superconductivity J. Polymer Sci.: Part C 29 3-26

Ginzburg V L 1976 High-temperature superconductivity-dream or reality? Usp. Fiz. Nauk 118 315-324 [1976 Sov. Phys. Usp. 19 174-179]

Ginzburg V L 1982, in High-Temperature Superconductivity ed V L Ginzburg and D A Kirznits (New York: Consultants Bureau) (translated from Russian)

[87] Ginzburg V L and Kirznits D A 1964 Zh. Eksper. Teor. Fiz. 46397 [1964 Sov. Phys.-JETP $19269]$

Ginzburg V L 1964 Zh. Eksper. Teor. Fiz. 472318 [1965 Sov. Phys.-JETP 20 1549]

Ginzburg V L 1964 Phys. Lett. 13101

[88] Akhiezer A I and Pomeranchuk I Ya 1959 Zh. Eksper. Teor. Fiz. 36859 [1959 Sov. Phys.-JETP 36 605-607]

[89] Paretti J 1962 Phys. Lett. 2275

Kondo J 1963 Superconductivity in transition metals Prog. Theor. Phys. 29 1-9

Privorotsky I A 1963 Zh. Eksper. Teor. Fiz. 44, 1401 (1963) [1963 Sov. Phys.-JETP 17 942]

Garland J W 1963 Phys. Rev. Lett. 11 111-114

Akhiezer A I and Akhiezer I A 1962 Zh. Eksper. Teor. Fiz. 432208

Vonsovsky S V and Svirsky 1964 Zh. Eksper. Teor. Fiz. 471354 [1965 Sov. Phys.-JETP 20 914]

Geilikman B T 1966 Usp. Fiz. Nauk 88327 [1966 Sov. Phys. Usp. 9 142]

Jensen M A and Suhl H 1966 Magnetism and superconductivity, in Magnetism ed G T Rado and H Suhl (San Diego: Academic Press) vol I, pp 215-291

[90] Matthias B T 1964 Science 144 138-141

[91] Ginzburg V L 1991 High-temperature superconductivity (history and general review) Usp. Fiz. Nauk 161 1-11 [1991 Sov. Phys. Usp. 34 283-288]

Ginzburg V L 1989 Prog. Low. Temp. Phys. 121

[92] Anderson P W 1994 A Career in Theoretical Physics (Singapore: World Scientific) pp 637-656, p 570, p 584

[93] Lee Y S, Birgeneau R J, Kastner M A, Endoh Y, Wakimoto S, Yamada K, Erwin R W, Lee S-H and Shirane G 1999 Phys. Rev. B 603642

Lee Y S, Birgeneau R J, Kastner M A, Endoh Y, Wakimoto S, Yamada K, Erwin R W, Lee S-H 
and Shirane G 1999 Preprint cond-mat/9902157

[94] Savici A T, Fudamoto Y, Gat I M, Ito T, Larkin M I, Uemura Y J, Luke G M, Kojima K M, Lee Y S, Kastner M A, Birgeneau R G and Yamada K 2000 Preprint cond-mat/0202037

[95] Ramires A P 1999 Nature 399527

Bozovic I, Logvenov G, Verhoeven M A J, Caputo P, Goldobin E and Geballe T H 2003 No mixing of superconductivity and antiferromagnetism in a high-temperature superconductor Nature $422873-5$

[96] Kirkpatrick T R and Belitz D 2003 Coexistence of ferromagnetism and superconductivity Phys. Rev. B 67024515

Karchev N 2003 Magnon exchange mechanism of ferromagnetic superconductivity Phys. Rev. B 67054416

Karchev N I, Blagoev K B, Bedell K S and Littlewood P 2001 Coexistence of superconductivity and ferromagnetism in ferromagnetic metals Phys. Rev. Lett. 86 846-849

Powell B J, Annett J F and Györffy 2003 J. Phys.: Condens. Matter 15 L235-L241

[97] Overhauser A W 1962 Phys. Rev. 128 1437-1452

[98] Presland M R et al. 1991 Physica C 17695

[99] Proust C, Boaknin E, Hill R W, Taillefer L and Mackenzie A P 2002 Phys. Rev. Lett. 89 147003,

Proust C, Boaknin E, Hill R W, Taillefer L and Mackenzie A P 2002 Heat transport in a strongly overdoped cuprate: Fermi liquid and pure d-wave BCS superconductor Preprint cond-mat/0202101

[100] Landau L D 1956 Zh. Eksp. Teor. Fiz 301058 [1957 Sov. Phys.-JETP 3 920-925]

Landau L D 1957 Zh. Eksp. Teor. Fiz 3259 [1957 Sov. Phys.-JETP 5 101]

[101] Anderson P W 2000 Physica C 341-348 9-10

[102] Pines D and Nozières Ph 1966 The Theory of Quantum Liquids vol 1 Normal Fermi Liquids (San Francisco: Benjamin)

[103] Kadanoff L P 2000 Statistical Physics. Statics, Dynamics and Renormalization (Singapore: World Scientific) p 128

[104] Landau L D 1941 J. Phys. USSR 571

[105] Anderson P W 1963 Concepts in Solids (San Francisco: Benjamin)

[106] Feng D L, Armitage N P, Lu D H, Damascelli A, Hu J P, Bogdanov P, Lanzara A, Ronning F, Shen K M, Eisaki H, Kim C, Shen Z-X, Shimoyama J-i and Kishio K 2001 Phys. Rev. Lett. 865550

[107] Anderson P W 1995 A battery of smoking guns (for the NFL-interlayer theory of high- $T_{\mathrm{C}}$ cuprates) J. Phys. Chem. Solids 56 1593-1596

[108] Hussey N E, Abdel-Jawad M, Carrington A, Mackenzie A P and Balicas L 2003 Observation of a coherent three-dimensional surface in a high- $T_{\mathrm{c}}$ superconductor Private communication (unpublished)

[109] Mishonov $\mathrm{T} 1991$ Predicted plasma oscillations in $\mathrm{Bi}_{2} \mathrm{Sr}_{2} \mathrm{CaCu}_{2} \mathrm{O}_{8}$ high- $T_{\mathrm{c}}$ superconductor Phys. Rev. B 4412033

[110] Anderson P W 1998 c-axis electrodynamics as evidence for the interlayer theory of hightemperature superconductivity Science 279 1196-1198; See, however, reference [61].

[111] Tamasaku K, Nakamura Y and Uchida U 1992 Phys. Rev. Lett. 691455

[112] Buisson O and Doria M M 2001 Plasma modes in HTS superconductors within the anisotropic London model, in High- $T_{\mathrm{c}}$ Superconductors and Related Materials: Materials Science, Fundamental Properties, and Some Future Electronic Applications ed S-L Drechsler and T Mishonov (Dordrecht: Kluwer) p 215

[113] Mishonov T and Groshev A 1990 Plasmon excitations in Josephson arrays and thin superconducting layers Phys. Rev. Lett. 642199

[114] Buisson O, Xavier P and Richard J 1994 Phys. Rev. Lett. 733153

Buisson O 2001 Plasma modes in thin superconducting films, in High-T $T_{\mathrm{c}}$ Superconductors and Related Materials: Materials Science, Fundamental Properties, and Some Future Electronic Applications ed S-L Drechsler and T Mishonov (Dordrecht: Kluwer) p 229

[115] Pauling L 1940 Nature of the Chemical Bond (Cornell: Cornell University Press)

Pauling L 1987 Phys. Rev. Lett. 59 225-227

[116] Eyring H, Walter J and Kimball G E 1944 Quantum Chemistry (New York: John Wiley \& Sons) chap XII, p 212

[117] Goodenough J B 1963 Magnetism and the Chemical Bond (New York: Wiley Interscience)

[118] de Gennes P G 1998 Simple Views on Condensed Matter expanded edition (Singapore: World Scientific) p 27: The author writes: "Indeed one can generate an interesting attraction electron-electron coupling via magnetic excitations. But the double exchange picture predicts 
a ferromagnetic state at strong doping, which has never been seen in the cuprates - hence this idea failed". And further: "A third group, led by P. Anderson, believes that electrons in the cuprate form a 'Luttinger liquid' which is not a simple Fermi liquid. I like the aesthetics of this approach, but I do not have a deep understanding of it".

de Gennes P G 1987 C. R. Acad. Sci. 305345

de Gennes P G 1960 Phys. Rev. 118 141-154

[119] Gofron K, Campuzano J C, Abrikosov A A, Lindroos M, Bansil A, Ding H, Koelling D and Dabrowski B 1994 Observation of an "extended" Van Hove singularity in $\mathrm{YBa}_{2} \mathrm{Cu}_{4} \mathrm{O}_{8}$ by ultrahigh energy resolution angle-resolved photoemission Phys. Rev. Lett. 73 3302-3305

[120] Bogoliubov N N, Tolmachev V V and Shirkov D V 1959 A New Method in the Theory of Superconductivity (New York: Consultants Bureau)

[121] Mishonov T M and Mishonov M T 2000 Simple model for the linear temperature dependence of the electrical resistivity of layered cuprates Physica A 278 553-562

(Preprint cond-mat/0001031)

[122] Hussey N E 2003 The normal state scattering rate in high- $T_{\mathrm{c}}$ cuprates Eur. Phys. J. B 31 495-507

Sandeman K G and Schofield A J 2001 Measuring anisotropic scattering in the cuprates Phys. Rev. B 63094510

Sandeman K G and Schofield A J 2000 Preprint cond-mat/0007299

[123] Kaminski A, Fretwell H M, Norman M R, Randeria M, Rosenkranz S, Chatterjee U, Campuzano J C, Mesot J, Sato T, Takahashi T, Terashima T, Takano M, Kadowaki K, Li Z Z and H. Raffy 2005, Phys. Rev. B $\mathbf{7 1} 014517$

[124] Yusof Z M, Wells B O, Valla T, Fedorov A V, Jonson P D, Li Q, Kendziora C, Jian Sha, Hinks D G 2002 Phys. Rev. Lett. 88167006

[125] Ziman J M 1972 Principles of the Theory of Solids 2nd edition (Cambridge: Cambridge University Press) p 336

[126] Abrikosov A A 1988 Fundamentals of the Theory of Metals (Amsterdam: North-Holland)

[127] Zaanen J 2002 Nature 415379

[128] Zaanen J 2000 Nature 404714

Coleman P 1998 Nature 392134

[129] Hudson E W, Lang K M, Madhavan V, Pan S H, Eisaki H, Uchida S and Davis J C 2001 Nature 411920

[130] Gonzalez Gonzalez J C, Albino Aguiar J, Quezado S, Dezanetie L M, Chu C W and Bustamante Dominguez A 2001 Physica C 354 441-443

[131] Carbotte J P, Schachinger E and Basov D N 1999 Nature 401 354-356

[132] Powell B J, Annett J F and Györffy B L 2002 Preprint cond-mat/0203087

[133] Mathur N D, Groshe F M, Jullian S R, Walker I R, Freye D M, Haselwimmer R K W and Lonzarich G G 1998 Nature 39439

Jordan M, Huth M and Adrian H 1999 Nature 39847

Saxena S S, Agarwal P, Ahilan K, Grosche F M, Haselwimmer R K W, Steiner M J, Pugh E, Walker I R, Julian S R, Monthoux P, Lonzarich G G, Huxley A, Sheikin I, Braithwaite D and Flouquet J 2000 Nature 406 587-592

Coleman P 2000 Nature 406580

[134] Farkas C, Brothwell S, Barnwell J and Fleishman S 2000 American Superconductor (Merrill Lynch, In-depth Report RC\#60133602)

[135] Bozovic I 2001 Atomic-layer engineering of superconducting oxides: Yesterday, today, tomorrow IEEE Trans. Appl. Supercond. 11 2686-2695

[136] Anderson P W 2000 Brainwashed by Feynman? Phys. Today 53 11-12

[137] Rosner H 1999 Electronic Structure and Exchange Integrals of Low-Dimensional Cuprates $P h D$ thesis Technische Universität Dresden, Germany

[138] Pajda M, Kudrnovský J, Turek I, Drchal V and Bruno P 2001 Phys. Rev. B 64174402

[139] Mishonov T M, Penev E S, Indekeu J O and Pokrovsky V L 2003 Specific heat discontinuity in impure two-band superconductors Phys. Rev. B 68, 104517

(Preprint cond-mat/0209342)

[140] Mishonov T M, Klenov S I and Penev E S 2005 Temperature dependence of specific heat and penetration depth of anisotropic-gap Bardeen-Cooper-Schrieffer superconductors for a factorizable pairing potential Phys. Rev. B 71, 024520

Mishonov T M, Klenov S I and Penev E S 2002 Preprint cond-mat/0212491

[141] Mishonov T, Drechsler S-L and Penev E 2003 Influence of the Van Hove singularity on the specific heat jump in BCS superconductors Mod. Phys. Lett. B 17 755-762

(Preprint cond-mat/0209192) 
[142] Bouvier J and Bok J 1998 Superconductivity in cuprates, the Van Hove scenario: a review, in The Gap Symmetry and Fluctuations in HTSC ed J Bok et al (New York: Plenum Press) p 37

[143] Mishonov T and Penev E 2002 Thermodynamics of anisotropic-gap and multiband clean BCS superconductors Int. J. Mod. Phys. B 16 3573-3586

(Preprint cond-mat/0206118)

[144] Mishonov T and Penev E 2000 Thermodynamics of Gaussian fluctuations and paraconductivity in layered superconductors Int. J. Mod. Phys. B 14 3831-3879

(Preprint cond-mat/0004023)

[145] Mishonov T and Penev E 2003 Bernoulli potential, Hall constant and Cooper pairs effective masses in disordered BCS superconductors Int. J. Mod. Phys. B 17 2883-2895

(Preprint cond-mat/0302168)

[146] Mishonov T M 1994 Technique for measuring the Cooper-pair velocity, density, and mass using Doppler splitting of the plasmon resonance in low-dimensional superconductor microstructures Phys. Rev. B 50 4004-4008

Mishonov T M 1994 Theory of Cooper-pair mass-spectroscopy by the current-induced contactpotential difference Phys. Rev. B 50 4009-4014

Mishonov T M 2000 In search for the vortex charge and the Cooper pair mass Superconducting and Related Oxides: Physics and Nanoengineering IV (SPIE Proc. 4058) ed D Pavuna and I Bozovic (Bellingham, WA: SPIE) pp 97-108

Mishonov T M 2000 Preprint cond-mat/0004286

Mishonov T, Nikolova D, Kaloyanov K and Klenov S 2001 Bernoulli effect in the quantum Hall regime and type-II superconductors in magnetic field, in High-T $T_{\mathrm{C}}$ Superconductors and Related Materials: Materials Science, Fundamental Properties, and Some Future Electronic Applications ed S-L Drechsler and T Mishonov (Dordrecht: Kluwer) pp 505-518

[147] Mishonov T M 1991 Comment on "Metallic and superconducting surfaces of $\mathrm{YBa}_{2} \mathrm{Cu}_{3} \mathrm{O}_{7}$ probed by electrostatic charge modulation of epitaxial films" Phys. Rev. Lett. 673195

[148] Mishonov T M and Zahariev N I 1999 Predicted new electrodynamic effect for thin films of layered cuprates - surface Hall currents induced by electric induction $D_{\perp}$ Superlatt. Microstruct. 26 57-60

[149] Pavarini E, Dasgupta I, Saha-Dasgupta T, Jepsen O and Andersen O K 2001 Band-Structure Trend in Hole-Doped Cuprates and Correlation with $T_{\mathrm{C} \text { max }}$, Phys. Rev. Lett. 87, 047003

[150] Mishonov T M 2004 Comment on "Band-Structure Trend in Hole-Doped Cuprates and Correlation with $T_{\mathrm{c}}$ max" Preprint cond-mat/0406357

[151] Stoll E P, Meier P F and Claxton T A 2003, J. Phys.: Condens. Matter 15, 7881

[152] Mishonov T M and Stoev M 2005 LCAO model for 3D Fermi surface of high- $T_{\mathrm{c}}$ cuprate $\mathrm{Tl}_{2} \mathrm{Ba}_{2} \mathrm{CuO}_{6+\delta}$ Preprint cond-mat/0504290 